\begin{tabular}{|c|l|}
\hline Title & Coherent shear phonon generation and detection with picosecond laser acoustics \\
\hline Author(s) & Matsuda, O.; Wright, O. B.; Hurley, D. H.; Gusev, V.; Shimizu, K. \\
\hline Citation & $\begin{array}{l}\text { Physical Review. B, Condensed Matter and Materials Physics, 77(22), 224110 } \\
\text { https://doi.org/L0.1103/PhysRevB.77.224110 }\end{array}$ \\
\hline Issue Date & 2008-06-20 \\
\hline Doc URL & http://hdl.handle.net/2115/34119 \\
\hline Rights & ○ 2008 A merican Physical Society \\
\hline Type & article \\
\hline File Information & Matsuda_prb2008.pdf \\
\hline
\end{tabular}

Instructions for use 


\title{
Coherent shear phonon generation and detection with picosecond laser acoustics
}

\author{
O. Matsuda and O. B. Wright \\ Division of Applied Physics, Graduate School of Engineering, Hokkaido University, Sapporo 060-8628, Japan
}

D. H. Hurley
Idaho National Laboratory, Idaho Falls, Idaho 83415-2209, USA

V. Gusev

Laboratoire de Physique de l'Etat Condensé, UMR CNRS 6087, Université du Maine, Av. O. Messiaen, 72085 Le Mans, France

K. Shimizu

University Chemical Laboratory, Keio University, Yokohama, Kanagawa 223-8521, Japan

(Received 19 November 2007; published 20 June 2008)

\begin{abstract}
Measurements on a film of silica on crystalline zinc using picosecond laser acoustics are theoretically analyzed to quantitatively explain the generation and detection of picosecond shear and longitudinal-acoustic waves. The theory encompasses the scattering of obliquely incident probe light of arbitrary polarization by a depth-dependent anisotropic permittivity modulation in a multilayer, including terms arising from the photoelastic effect, interface displacements, and local rotations. Sound velocities, ultrasonic attenuation, and photoelastic constants are experimentally derived.
\end{abstract}

DOI: 10.1103/PhysRevB.77.224110

PACS number(s): 92.60.Ta, 78.35.+c, 78.47.jc, 78.20.Hp

\section{INTRODUCTION}

The absorption of ultrashort light pulses in a medium will generate picosecond acoustic pulses therein. ${ }^{1}$ The acoustic propagation can be measured with ultrashort light pulses through transient reflectivity changes or surface displacements caused by the acoustic strain field in the medium. This technique, known as picosecond laser acoustics or picosecond ultrasonics, has been widely used for the nondestructive testing of thin metal, semiconductor, and dielectric films and nanostructures. ${ }^{1-12}$

Studies with picosecond laser acoustics on thin isotropic films of micron or nanometer scale thickness produce only longitudinal-acoustic waves in the in-depth direction owing to the symmetry of the generation conditions. However, the range of measurable physical properties can be greatly increased by the use of shear waves. With this goal in mind, material anisotropy ${ }^{13-17}$ and three-dimensional propagation involving mode conversion ${ }^{18,19}$ have been recently exploited to optically generate and detect picosecond shear waves. For the generation of high-frequency shear waves in thin films, the use of material anisotropy is preferable because this method generates plane shear waves propagating perpendicular to the sample surface. Shear waves are generated in this case by the use of crystals with a surface cut in a plane off the axes of crystal symmetry. A rigorous quantitative analysis of the detection theory for such geometries is, however, lacking. In previous related work ${ }^{17}$ involving a $\mathrm{Zn}$ substrate coated with a $\mathrm{ZnO}$ film, the transient reflectivity change caused by the strain field was analyzed for normally incident probe light without accounting for the multiple optical reflections in the film. Only the ratio of the photoelastic tensor components for $\mathrm{Zn}$, rather than the absolute values, could be derived.

In this paper, we present a theory of light scattering appropriate for shear strain detection in thin films and multi- layers and apply it to experimental results for picosecond shear acoustic generation and detection in a $\mathrm{SiO}_{2} / \mathrm{Zn}$ sample. The theory handles obliquely incident probe light and accounts for the effects of the multiple optical reflections in the multilayer structure. We demonstrate that the former feature is essential for shear wave detection in isotropic media, and that the latter feature allows the determination of absolute values of the photoelastic constants.

We first present the theory of generation, propagation, and detection of longitudinal and shear acoustic waves. The detection theory is based on a Green's function formalism applicable to multilayers. Then we describe the experimental results and compare them with theory. We present the theory in a general form for multilayers, while its demonstration in the analysis of the experimental results is kept as simple as possible. Details of the theory, such as the derivation of the Green's function, are described in Appendixes A-C.

\section{THEORY}

\section{A. Background}

Picosecond laser acoustics is typically applied to multilayers whose layer thicknesses are of nanometer to micron order. The generation (pump) light pulses are usually focused on the sample surface with a spot diameter $D_{p u}$ of $\sim 10-100 \mu \mathrm{m}$. In this case, the generated acoustic disturbance can be approximated as a superposition of plane waves propagating in the depth $(z)$ direction for propagation distances small compared to the acoustic diffraction length $D_{p u}^{2} / \lambda$, where $\lambda$ is the characteristic acoustic wavelength. In this paper, we consider samples in which laterally homogeneous thin layers are stacked in the $z$ direction and assume that the modulation caused by the acoustic wave depends only on $z$. 


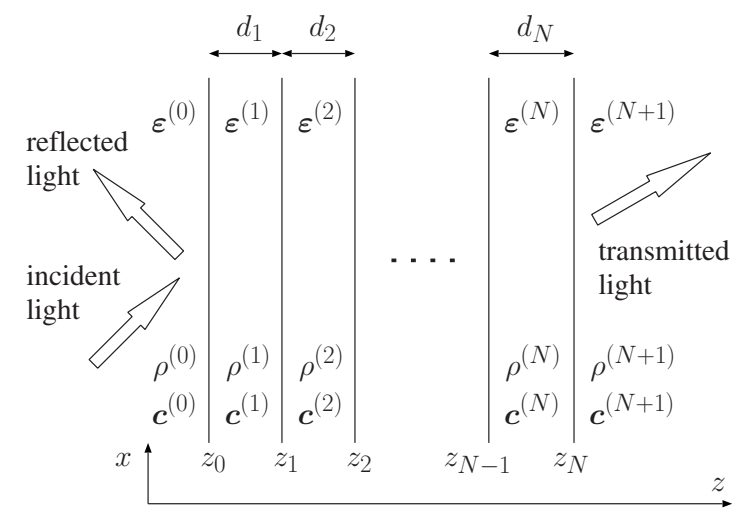

FIG. 1. $N$ layers on a substrate. The incident light comes from $z<0$. Each layer is assumed to be homogeneous in its equilibrium state. $\boldsymbol{\varepsilon}^{(n)}$, permittivity; $\boldsymbol{\rho}^{(n)}$, mass density; and $\boldsymbol{c}^{(n)}$, elastic stiffness of $n$th layer.

In the geometry we consider, the detection (probe) light pulses are focused normally or obliquely onto the sample surface with a spot size that is the same or somewhat smaller than that of the pump light pulses. Within the focal depth of the focused probe light we may regard the probe light as a plane wave. As will be discussed in Sec. IV A, the use of oblique probe incidence allows the detection of shear waves propagating normal to the stacking direction in isotropic media. With these assumptions, both the electromagnetic and the acoustic waves can be treated as plane waves. This is the basis of the detection analysis described below.

\section{B. Light scattering in inhomogeneously modulated multilayers}

Consider the light scattering caused by an inhomogeneous depth-dependent modulation in a multilayer. ${ }^{54}$ General optical polarization at oblique optical incidence is considered. The medium itself and the modulation in refractive index may be anisotropic. The theory is based on an extension of previous work for normally incident light. ${ }^{3,20,21}$

Consider the multilayer shown in Fig. 1, consisting of $N$ layers on a substrate. The sample surface is located at $z=z_{0}$ $=0$ when at rest, and the region $z<0$ is a vacuum or filled with a uniform medium (air, glass, etc.). The $n$th layer has thickness $d_{n}$ and the interface between the $n$th and $(n+1)$ th layer is at $z_{n}$. The final layer-the $(N+1)$ th layer-is taken as an infinite substrate (or possibly a surrounding medium). The sample and surroundings have translational symmetry in the $x-y$ plane. Each layer and the substrate are homogeneous in the equilibrium state and may be transparent or opaque and anisotropic or isotropic.

The incident light is assumed to be a monochromatic plane wave with angular frequency $\omega=c k$, where $k$ is the vacuum wave number. The light may have arbitrary polarization ( $p, s$, circular, etc.). The $x$ axis is taken so that the plane of optical incidence is parallel to $x-z$ plane so that the $x$ components of the incident light wave vector $k_{x}$ are positive. Due to the translational symmetry in the $x-y$ plane, the electromagnetic field depends on $x$ according to $\exp \left(i k_{x} x\right)$ everywhere.
The sample (and the surrounding medium) is perturbed by the propagating acoustic wave, whose accompanying strain field modulates the dielectric permittivity of the sample through the photoelastic effect. Because we only consider acoustic plane waves propagating in the $z$ direction, the modulation in permittivity $\Delta \varepsilon$ depends only on $z$. Since this permittivity modulation may involve arbitrary anisotropy (i.e., involving birefringence), $\Delta \boldsymbol{\varepsilon}$ should be expressed in tensor form. The modulation is assumed to occur on a time scale that is much slower than the optical period so that quasistatic theories can be applied and no optical frequency conversion occurs. Since an acoustic wave is a wave of elastic displacement, the displacement of the surface or interfaces in the $z$ direction must also be considered and can be expressed as a modulation in $\Delta \varepsilon$ in the near-surface or nearinterface regions. ${ }^{3,21}$ In addition, the local rotation associated with shear acoustic waves contributes to $\Delta \varepsilon$ in anisotropic media. Using the above assumptions we shall derive the change in (complex) reflectance and transmittance (provided some light is transmitted) caused by $\Delta \boldsymbol{\varepsilon}(z)$.

From Maxwell's equations, the general wave equation for a monochromatic electric field $\boldsymbol{E}(\boldsymbol{r}) \exp (-i \omega t)$ is given by ${ }^{55}$

$$
\operatorname{grad} \operatorname{div} \boldsymbol{E}-\nabla^{2} \boldsymbol{E}=\mu_{0} \omega^{2} \boldsymbol{D},
$$

where $\boldsymbol{D}(\boldsymbol{r}) \exp (-i \omega t)$ is the electric displacement field. In the linear-response regime,

$$
D=\varepsilon_{0} \varepsilon E,
$$

where the relative permittivity tensor $\boldsymbol{\varepsilon}(\boldsymbol{r})$ may depend on the position $\boldsymbol{r}$. The wave equation then becomes

$$
\left(\nabla^{2}-\operatorname{grad} \operatorname{div}+k^{2} \boldsymbol{\varepsilon}\right) \boldsymbol{E}=0 .
$$

The lateral homogeneity of the sample allows further simplification. Since the optical plane of incidence is the $x-z$ plane, the electric field is expressed as $\boldsymbol{E}(z) \exp \left\{i\left(k_{x} x-\omega t\right)\right\}$. Equation (3) can be converted to

$$
\left\{\boldsymbol{L}\left(k_{x}\right)+k^{2} \boldsymbol{\varepsilon}(z)\right\} \boldsymbol{E}(z)=0,
$$

where the operator $\boldsymbol{L}\left(k_{x}\right)$ is defined as

$$
\boldsymbol{L}\left(k_{x}\right) \equiv\left(\begin{array}{ccc}
\frac{d^{2}}{d z^{2}} & 0 & -i k_{x} \frac{d}{d z} \\
0 & \frac{d^{2}}{d z^{2}}-k_{x}^{2} & 0 \\
-i k_{x} \frac{d}{d z} & 0 & -k_{x}^{2}
\end{array}\right) .
$$

This is the equation that we shall solve.

The position dependent permittivity can be divided into two parts, a homogeneous part $\boldsymbol{\varepsilon}_{h}(z)$ and an inhomogeneous part $\Delta \varepsilon(z)$,

$$
\boldsymbol{\varepsilon}(z)=\boldsymbol{\varepsilon}_{h}(z)+\Delta \boldsymbol{\varepsilon}(z) .
$$

The homogeneous part is a staircaselike function of $z$ representing the permittivity for each layer in the equilibrium state of the piecewise homogeneous sample, 


$$
\boldsymbol{\varepsilon}_{h}(z)=\boldsymbol{\varepsilon}^{(n)} \text { for } z \in\left(z_{n-1}, z_{n}\right),
$$

with $z_{-1}=-\infty$ and $z_{N+1}=+\infty$. The inhomogeneous part arises from the propagating acoustic waves and will be discussed in detail in Sec. II D.

Suppose that we know the solution $\boldsymbol{E}_{0}$ of the wave equation for our piecewise homogeneous sample,

$$
\left\{\boldsymbol{L}\left(k_{x}\right)+k^{2} \boldsymbol{\varepsilon}_{h}(z)\right\} \boldsymbol{E}_{0}(z)=0,
$$

as well as the $3 \times 3$ matrix of the Green's function $\boldsymbol{G}\left(z, z^{\prime}\right)$, satisfying

$$
\left\{\boldsymbol{L}\left(k_{x}\right)+k^{2} \boldsymbol{\varepsilon}_{h}(z)\right\} \boldsymbol{G}\left(z, z^{\prime}\right)=-\delta\left(z-z^{\prime}\right) \boldsymbol{I},
$$

where $\boldsymbol{I}$ is the $3 \times 3$ identity matrix. $\boldsymbol{E}_{0}$ is the solution for the unperturbed medium, made up of a sum of incident, reflected, and transmitted light fields. The solution $\boldsymbol{E}$ for the wave equation with an arbitrary inhomogeneous component,

$$
\left\{\boldsymbol{L}\left(k_{x}\right)+k^{2}\left[\boldsymbol{\varepsilon}_{h}(z)+\Delta \boldsymbol{\varepsilon}(z)\right]\right\} \boldsymbol{E}(z)=0,
$$

can be rigorously expressed as ${ }^{22,23}$

$$
\boldsymbol{E}(z)=\boldsymbol{E}_{0}(z)+k^{2} \int_{-\infty}^{\infty} d z^{\prime} \boldsymbol{G}\left(z, z^{\prime}\right) \Delta \boldsymbol{\varepsilon}\left(z^{\prime}\right) \boldsymbol{E}\left(z^{\prime}\right) .
$$

When $\Delta \boldsymbol{\varepsilon}$ is small, Eq. (11) can be expanded as

$$
\begin{aligned}
\boldsymbol{E}(z) \simeq & \boldsymbol{E}_{0}(z)+k^{2} \int_{-\infty}^{\infty} d z^{\prime} \boldsymbol{G}\left(z, z^{\prime}\right) \Delta \boldsymbol{\varepsilon}\left(z^{\prime}\right) \boldsymbol{E}_{0}\left(z^{\prime}\right) \\
& +k^{4} \int_{-\infty}^{\infty} d z^{\prime} \int_{-\infty}^{\infty} d z^{\prime \prime} \boldsymbol{G}\left(z, z^{\prime}\right) \Delta \boldsymbol{\varepsilon}\left(z^{\prime}\right) \boldsymbol{G}\left(z^{\prime}, z^{\prime \prime}\right) \\
& \times \Delta \boldsymbol{\varepsilon}\left(z^{\prime \prime}\right) \boldsymbol{E}_{0}\left(z^{\prime \prime}\right)+\cdots
\end{aligned}
$$

Here $\boldsymbol{G}$ and $\Delta \boldsymbol{\varepsilon}$ are matrices and are thus not commutative. When $\Delta \varepsilon$ is small, Eq. (12) can be truncated to first order with high accuracy. Any disturbance other than strain that modulates the permittivity, such as temperature changes or excited electron distributions, can be treated in the same manner. The derivation of $\boldsymbol{E}_{0}$ and $\boldsymbol{G}$ for our multilayer geometry are given in Appendixes A and B.

\section{Acoustic wave generation and propagation in multilayers}

In this section we describe the generation and propagation of acoustic waves in our anisotropic multilayer. ${ }^{24,25} \mathrm{We}$ present the essential details required to calculate the inhomogeneous perturbation in strain. As discussed in Sec. II A, the acoustic source is assumed to be laterally homogeneous. As a consequence of this the acoustic waves propagate only in the $z$ direction, and the acoustic field depends only on the $z$ coordinate. We first consider the thermoelastic effect in the absence of diffusion processes as the origin of the acoustic source. The displacement field $\boldsymbol{u}(z, t)$ for $z$ lying within the $n$th layer satisfies the acoustic wave equation,

$$
\rho^{(n)} \frac{\partial^{2} u_{i}}{\partial t^{2}}=\frac{\partial \sigma_{i z}}{\partial z},
$$

$$
\sigma_{i z}=c_{i z k l}^{(n)}\left[\eta_{k l}-\alpha_{k l}^{(n)} \Delta T(z, t)\right],
$$

where $\sigma_{i j}$ is the stress field, $\eta_{i j}$ is the strain field, and $\Delta T$ is the transient temperature rise. Also $\rho^{(n)}, c_{i j k l}^{(n)}, \alpha_{i j}^{(n)}$ are the mass density, elastic stiffness, and thermal-expansion coefficient of the $n$th layer, respectively. The summation convention for repeated subscripts applies to Eq. (13) and subsequent equations, unless otherwise stated.

We further simplify the problem by assuming a stepwise temperature rise at $t=0: \Delta T(z, t)=\Delta T(z) \theta(t)$, where $\theta(t)$ is the Heaviside function. The initial condition is $\left.\boldsymbol{u}(z, t)\right|_{t=0}$ $=0$ and $\partial \boldsymbol{u}(z, t) /\left.\partial t\right|_{t=0}=0$ throughout the medium. We can express the solution of Eq. (13) for $t>0$ as the sum of time dependent and time independent parts,

$$
\boldsymbol{u}(z, t)=\boldsymbol{u}_{A}(z, t)+\boldsymbol{u}_{B}(z) .
$$

The displacements $\boldsymbol{u}_{A}$ and $\boldsymbol{u}_{B}$ are obtained from the solutions of

$$
\rho^{(n)} \frac{\partial^{2} u_{A i}}{\partial t^{2}}=c_{i z j z}^{(n)} \frac{\partial^{2} u_{A j}}{\partial z^{2}},
$$

$$
0=c_{i z j z}^{(n)} \frac{\partial^{2} u_{B j}}{\partial z^{2}}-c_{i z k l}^{(n)} \alpha_{k l}^{(n)} \frac{d \Delta T(z)}{d z} .
$$

Physically $\boldsymbol{u}_{B}$ corresponds to the final equilibrium state, whereas $\boldsymbol{u}_{A}$ corresponds to the propagating wave relative to the final state.

Equation (15a) leads to three acoustic modes with velocity $v_{\mu}^{(n)}$ and displacement polarization $\boldsymbol{u}_{\mu}^{(n)}$, where $\mu=1,2$, and 3 is the mode label. These are obtained as the eigenvalues and eigenvectors of

$$
\boldsymbol{\rho}^{(n)}\left\{v_{\mu}^{(n)}\right\}^{2} \boldsymbol{u}_{\mu}^{(n)}=\boldsymbol{C}^{(n)} \boldsymbol{u}_{\mu}^{(n)},
$$

where $\boldsymbol{C}^{(n)}$ is a $3 \times 3$ matrix defined as

$$
\boldsymbol{C}^{(n)} \equiv\left(\begin{array}{ccc}
c_{55}^{(n)} & c_{54}^{(n)} & c_{53}^{(n)} \\
c_{45}^{(n)} & c_{44}^{(n)} & c_{43}^{(n)} \\
c_{35}^{(n)} & c_{34}^{(n)} & c_{33}^{(n)}
\end{array}\right)
$$

The abbreviated index notation $c_{I J}$ for elastic constants is used instead of $c_{i j k l}$ here. The summation convention for $\mu$ does not apply to the left-hand side of Eq. (16). The general solution for Eq. (15a) is given by

$$
\boldsymbol{u}_{A}^{(n)}(z, t)=\boldsymbol{u}_{\mu}^{(n)}\left\{f_{\mu}^{(n)}\left(z-v_{\mu}^{(n)} t\right)+g_{\mu}^{(n)}\left(z+v_{\mu}^{(n)} t\right)\right\} .
$$

Here $f_{\mu}^{(n)}(\xi)$ and $g_{\mu}^{(n)}(\xi)$ are arbitrary functions.

It is convenient to introduce a column vector

$$
\boldsymbol{\eta} \equiv\left(\begin{array}{c}
\eta_{x} \\
\eta_{y} \\
\eta_{z}
\end{array}\right) \equiv\left(\begin{array}{c}
\partial u_{x} / \partial z \\
\partial u_{y} / \partial z \\
\partial u_{z} / \partial z
\end{array}\right)=\left(\begin{array}{c}
2 \eta_{x z} \\
2 \eta_{y z} \\
\eta_{z z}
\end{array}\right)=\left(\begin{array}{c}
\eta_{5} \\
\eta_{4} \\
\eta_{3}
\end{array}\right) .
$$

Note that $\eta_{x}, \eta_{y}$, and $\eta_{z}$ should not be confused with the abbreviated index notation of strain; $\eta_{1}=\eta_{x x}, \eta_{2}=\eta_{y y}, \eta_{3}$ $=\eta_{z z}, \eta_{4}=2 \eta_{y z}, \eta_{5}=2 \eta_{z x}$, and $\eta_{6}=2 \eta_{x y}$. Similarly $\boldsymbol{\eta}_{A}$ and $\boldsymbol{\eta}_{B}$ are defined as $\partial \boldsymbol{u}_{A} / \partial z$ and $d \boldsymbol{u}_{B} / d z$. Here $\boldsymbol{\eta}_{B}$ can be termed as a static strain: it is the nonpropagating component of the strain for a material containing a static thermal expansion. At 
$t=0$, the two strain components cancel and $\boldsymbol{\eta}_{A}+\boldsymbol{\eta}_{B}=0$. Equation (18) gives

$$
\boldsymbol{\eta}_{A}^{(n)}(z, t)=\boldsymbol{u}_{\mu}^{(n)}\left\{f_{\mu}^{(n)^{\prime}}\left(z-v_{\mu}^{(n)} t\right)+g_{\mu}^{(n)^{\prime}}\left(z+v_{\mu}^{(n)} t\right)\right\},
$$

where $f_{\mu}^{(n)^{\prime}}(\xi)=d f_{\mu}^{(n)}(\xi) / d \xi$ and $g_{\mu}^{(n)^{\prime}}(\xi)=d g_{\mu}^{(n)}(\xi) / d \xi$.

Because the strain should vanish when $\Delta T \rightarrow 0$, we find

$$
c_{i z j z}^{(n)} \eta_{B j}-c_{i z k l}^{(n)} \alpha_{k l}^{(n)} \Delta T(z)=0
$$

on integrating Eq. (15b) with respect to $z$. This is a set of linear simultaneous equations and can be solved for $\boldsymbol{\eta}_{B}$ for a given $\Delta T$. The quantity $c_{i z j z}^{(n)} \eta_{B j}=c_{i z k l}^{(n)} \alpha_{k l}^{(n)} \Delta T(z)$ at $t=0$ can be interpreted as the thermoelastic stress. To obtain $f_{\mu}^{(n)^{\prime}}(\xi)$ and $g_{\mu}^{(n)^{\prime}}(\xi)$, we express $\boldsymbol{\eta}_{B}$ as a linear combination of $\boldsymbol{u}_{\mu}^{(n)}$,

$$
\boldsymbol{\eta}_{B}(\xi)=\boldsymbol{u}_{\mu}^{(n)} h_{\mu}^{(n)}(\xi) .
$$

From initial condition for $\boldsymbol{u}$ and $\partial \boldsymbol{u} / \partial t$, one can show that

$$
f_{\mu}^{(n)^{\prime}}(\xi)=g_{\mu}^{(n)^{\prime}}(\xi)=-\frac{1}{2} h_{\mu}^{(n)}(\xi)
$$

for $\xi \in\left(z_{n-1}, z_{n}\right)$.

Equations (18) and (20) require $f_{\mu}^{(n)}(\xi)$, etc., to be defined for $\xi \in(-\infty, \infty)$, whereas Eqs. (21)-(23) allow these functions to be determined only for a limited range of $\xi$. To extend the domain for $f_{\mu}^{(n)^{\prime}}$ and $g_{\mu}^{(n)^{\prime}}$, we require the reflection and transmission coefficients for the propagating strain field accounting for the effects of mode conversion. At each interface, the stress components $\sigma_{x z}, \sigma_{y z}$, and $\sigma_{z z}$ and displacement $u_{x}, u_{y}$, and $u_{z}$ should be continuous. The continuity in the displacement also implies the continuity in the time derivative of the displacement. The boundary condition can thus be expressed as

$$
\begin{aligned}
& v_{\mu}^{(n)} \boldsymbol{u}_{\mu}^{(n)}\left\{f_{\mu}^{(n)^{\prime}}\left(z_{n}-v_{\mu}^{(n)} t\right)-g_{\mu}^{(n)^{\prime}}\left(z_{n}+v_{\mu}^{(n)} t\right)\right\} \\
& =v_{\nu}^{(n+1)} \boldsymbol{u}_{\nu}^{(n+1)}\left\{f_{\nu}^{(n+1)^{\prime}}\left(z_{n}-v_{\nu}^{(n+1)} t\right)\right. \\
& \left.\quad-g_{\nu}^{(n+1)^{\prime}}\left(z_{n}+v_{\nu}^{(n+1)} t\right)\right\}, \\
& \boldsymbol{C}^{(n)} \boldsymbol{u}_{\mu}^{(n)}\left\{f_{\mu}^{(n)^{\prime}}\left(z_{n}-v_{\mu}^{(n)} t\right)+g_{\mu}^{(n)^{\prime}}\left(z_{n}+v_{\mu}^{(n)} t\right)\right\} \\
& =\boldsymbol{C}^{(n+1)} \boldsymbol{u}_{\nu}^{(n+1)}\left\{f_{\nu}^{(n+1)^{\prime}}\left(z_{n}-v_{\nu}^{(n+1)} t\right)\right. \\
& \left.+g_{\nu}^{(n+1)^{\prime}}\left(z_{n}+v_{\nu}^{(n+1)} t\right)\right\} .
\end{aligned}
$$

Equation (24) consists of six linear equations that allow $g_{\mu}^{(n)^{\prime}}\left(z_{n}+v_{\mu}^{(n)} t\right)$ and $f_{\mu}^{(n+1)^{\prime}}\left(z_{n}-v_{\mu}^{(n+1)} t\right)$ to be obtained from $f_{\mu}^{(n)^{\prime}}\left(z_{n}-v_{\mu}^{(n)} t\right)$ and $g_{\mu}^{(n+1)^{\prime}}\left(z_{n}+v_{\mu}^{(n+1)} t\right)$. In this way, the domain for strain propagation can be extended as much as one needs.

The procedure mentioned above is easily extended for general time dependence of $\Delta T(z, t)$. Assuming $\Delta T(z, t)=0$ for $t \leq 0$, the relation

$$
\Delta T(z, t)=\int_{0}^{\infty} \frac{\partial \Delta T(z, \tau)}{\partial \tau} \theta(t-\tau) d \tau
$$

holds. This implies that the time varying temperature rise can be regarded as an infinite set of stepwise temperature rises $\partial \Delta T / \partial t$ distributed in temporal domain. Each temperature rise at $t$ additively generates acoustic waves at $t$.

\section{Permittivity modulation by acoustic waves in multilayers}

The propagating acoustic waves modulate the permittivity of the medium $(\Delta \varepsilon)$ through the photoelastic effect (pe), the surface or interface displacement (if), and the local rotation (rt) according to

$$
\Delta \boldsymbol{\varepsilon}(z, t)=\Delta \boldsymbol{\varepsilon}^{(\mathrm{pe})}(z, t)+\Delta \boldsymbol{\varepsilon}^{(\mathrm{if})}(z, t)+\Delta \boldsymbol{\varepsilon}^{(\mathrm{rt})}(z, t) .
$$

The photoelastic contribution is proportional to the strain. Using the abbreviated index notation, it can be expressed as

$$
\Delta \varepsilon_{I}^{(\mathrm{pe})}(z, t)=P_{I J}^{(n)} \eta_{J}(z, t)
$$

where $n$ is the layer number in which $z$ resides. The coefficients $P_{I J}^{(n)}$ are components of the photoelastic tensor of the $n$th layer. ${ }^{26}$ This tensor can be expressed as a $6 \times 6$ matrix, but it is in fact a fourth rank tensor. In the case of anisotropic media, it also depends on the orientation of the crystal axes.

The displacement is caused by propagating acoustic waves as well as by the static thermal expansion. Because of the lateral homogeneity of the medium, the $x$ and $y$ components of the displacement have no effect on the effective permittivity. However, the displacement normal to the surface or interface $\left(u_{z}\right)$ is equivalent to one medium penetrating into the other. The effective change in relative permittivity can therefore be expressed as ${ }^{21}$

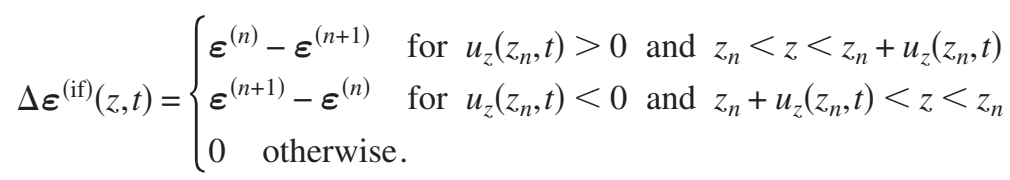


The local rotation is caused by the shear strain field. This rotation can be expressed by a $3 \times 3$ rotation matrix, ${ }^{26}$

$$
\begin{gathered}
R_{i j}=\delta_{i j}+w_{i j}, \\
w_{i j}=\frac{1}{2}\left\{\frac{\partial u_{i}}{\partial x_{j}}-\frac{\partial u_{j}}{\partial x_{i}}\right\} .
\end{gathered}
$$

The change in crystal orientation modulates the permittivity tensor according to ${ }^{27,28}$

$$
\varepsilon_{i j}+\Delta \varepsilon_{i j}^{(\mathrm{rt})} \simeq R_{i k} R_{j l} \varepsilon_{k l} \simeq \varepsilon_{i j}+w_{i k} \varepsilon_{k j}-\varepsilon_{i l} w_{l j} .
$$

or

$$
\Delta \varepsilon^{(\mathrm{rt})}=w \varepsilon-\varepsilon w
$$

The quantity $\Delta \varepsilon^{(\mathrm{rt})}$ vanishes when $\varepsilon$ is isotropic. In our particular geometry, the tensor $\boldsymbol{w}$ has the form

$$
\boldsymbol{w}=\frac{1}{2}\left(\begin{array}{ccc}
0 & 0 & \partial u_{x} / \partial z \\
0 & 0 & \partial u_{y} / \partial z \\
-\partial u_{x} / \partial z & -\partial u_{y} / \partial z & 0
\end{array}\right)=\frac{1}{2}\left(\begin{array}{ccc}
0 & 0 & \eta_{5} \\
0 & 0 & \eta_{4} \\
-\eta_{5} & -\eta_{4} & 0
\end{array}\right) .
$$

Substituting Eqs. (27), (28), and (31) into Eq. (26) and making use of Eq. (12), we can calculate the acoustically induced perturbation in electric field. The term involving the displacement contribution $\Delta \varepsilon^{(i f)}$ requires special care. Since $u_{z}$ is much smaller than the optical wavelength, the integral can be approximated as

$$
\begin{aligned}
\int_{-\infty}^{\infty} & d z^{\prime} \boldsymbol{G}\left(z, z^{\prime}\right) \Delta \boldsymbol{\varepsilon}^{(\mathrm{if})}\left(z^{\prime}\right) \boldsymbol{E}_{0}\left(z^{\prime}\right) \\
\simeq & \sum_{n=0}^{N} \boldsymbol{G}\left(z, z_{n}\right)\left[\boldsymbol{\varepsilon}^{(n)}-\boldsymbol{\varepsilon}^{(n+1)}\right] \boldsymbol{E}_{0}\left(z_{n}\right) u_{z}\left(z_{n}\right) .
\end{aligned}
$$

A problem arises when $\boldsymbol{G}\left(z, z_{n}\right)$ or $\boldsymbol{E}_{0}(z)$ has a discontinuity at $z=z_{n}$ : it is not clear which value, $z=z_{n}+0$ or $z=z_{n}-0$, should be used. These values of $z$ should be distinguished because of discontinuities in the $z$ component of the electric field and in the Green's function at the interfaces, a problem that does not arise for normal optical incidence. Since the solution is rigorously given by Eq. (11), and since Eq. (12) is obtained by approximating $\boldsymbol{E}$ in the integral with $\boldsymbol{E}_{0}$, one should choose $\boldsymbol{E}_{0}\left(z_{n}\right)$ to be closest to the expected solution $\boldsymbol{E}\left(z_{n}\right)$. Therefore, for $u_{z}\left(z_{n}\right)>0$ one should use $\boldsymbol{G}\left(z, z_{n}+0\right)$ and $\boldsymbol{E}_{0}\left(z_{n}-0\right)$, whereas for $u_{z}\left(z_{n}\right)<0$ one should use $\boldsymbol{G}\left(z, z_{n}-0\right)$ and $\boldsymbol{E}_{0}\left(z_{n}+0\right)$. However, we also know that the change in the electric field $\boldsymbol{E}(z)$ caused by the surface or interface displacements should be zero for $u_{z}\left(z_{n}\right)=0$, and also that these changes should be linear in the displacements $u_{z}\left(z_{n}\right)$. Therefore Eq. (33) can be evaluated in the following form:

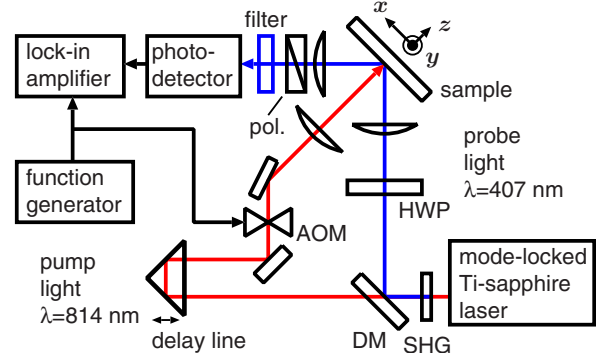

FIG. 2. (Color online) Experimental setup and definition of axes. SHG, second-harmonic generation crystal; HWP, half-wave plate; DM, dichroic mirror; pol., polarizer; and AOM, acousto-optic modulator.

$$
\begin{aligned}
\int_{-\infty}^{\infty} & d z^{\prime} \boldsymbol{G}\left(z, z^{\prime}\right) \Delta \boldsymbol{\varepsilon}^{(\mathrm{if})}\left(z^{\prime}\right) \boldsymbol{E}_{0}\left(z^{\prime}\right) \\
\simeq & \sum_{n=0}^{N} \boldsymbol{G}\left(z, z_{n}+0\right)\left[\boldsymbol{\varepsilon}^{(n)}-\boldsymbol{\varepsilon}^{(n+1)}\right] \boldsymbol{E}_{0}\left(z_{n}-0\right) u_{z}\left(z_{n}\right),
\end{aligned}
$$

even for the negative $u_{z}\left(z_{n}\right)$.

\section{EXPERIMENT AND RESULTS}

To demonstrate the proposed theory, we carry out a picosecond laser acoustics experiment with obliquely incident probe light on a relatively simple system consisting of an isotropic transparent film on an anisotropic substrate. The experiment involves the generation and detection of shear as well as longitudinal-acoustic waves.

A highly anisotropic $\mathrm{Zn}$ single crystal (of the hexagonal system) is used as an optoacoustic transducer. ${ }^{14}$ The crystal is cut with a surface $45^{\circ}$ off the (0001) plane. ${ }^{56}$ The mechanically polished surface is trimmed with a diamond knife using an ultramicrotome to remove a damaged and oxidized layer. ${ }^{29}$ The surface is then coated with a $\mathrm{SiO}_{2}$ film of nominal thickness $1.1 \mu \mathrm{m}$ by radio-frequency sputtering.

Figure 2 shows a schematic diagram of the experimental setup. Pump light pulses from a mode-locked Ti:sapphire laser with a repetition frequency of $76 \mathrm{MHz}$, a pulse duration of $400 \mathrm{fs}$, and a wavelength of $814 \mathrm{~nm}$ are focused onto the $\mathrm{SiO}_{2}$ coated sample surface with a spot diameter of $\sim 60 \mu \mathrm{m}$ and a fluence of $\sim 0.05 \mathrm{~mJ} \mathrm{~cm} \mathrm{~cm}^{-2}$ at normal incidence to generate the acoustic pulses. Probe light pulses from the same laser are frequency doubled with a $\beta-\mathrm{BaB}_{2} \mathrm{O}_{4}$ crystal and are focused onto the same region as the pump light with a spot diameter $\sim 20 \mu \mathrm{m}$ and fluence $\sim 0.005 \mathrm{~mJ} \mathrm{~cm}^{-2}$. The probe angle of incidence is $45^{\circ}$.

The $z$ axis is taken in the depth direction of the sample, and the $x$ axis lies in the incident plane of the probe light so that $k_{x}$ is positive (Fig. 2). The $c$ axis of the $\mathrm{Zn}$ crystal is directed along the vector $(0,1,1)$ in the $x y z$ space. In this case, no $\eta_{5}$ (i.e., $x z$ shear) component of strain is expected to be generated. 


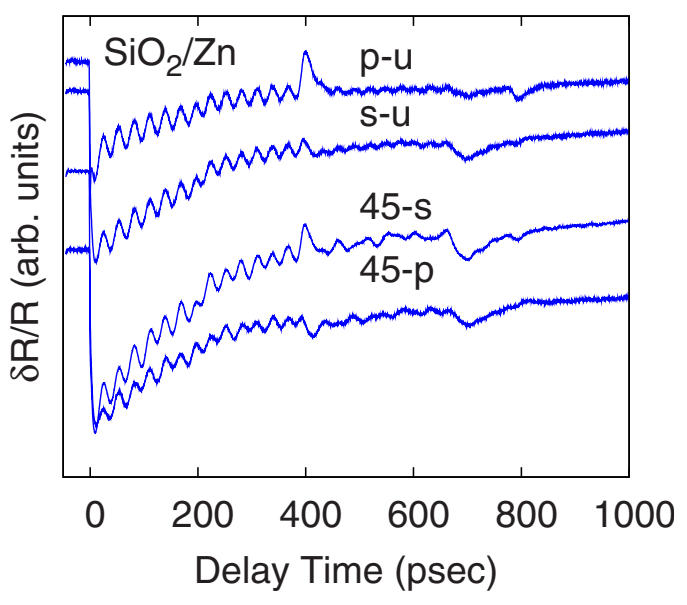

FIG. 3. (Color online) Raw data for the transient reflectivity change in the $\mathrm{SiO}_{2} / \mathrm{Zn}$ sample. The polarization of the incident light $\left(p, s\right.$, and $\left.45^{\circ}\right)$ and the reflected light $(p, s$, and $u)$ are specified on each curve. The scales are common for all curves, which are shifted vertically for clarity.

Using a half-wave plate, the incident probe light polarization is sequentially chosen as $p, s$, and at $45^{\circ}$ to the plane of incidence, corresponding to the polarization vectors $(1 / \sqrt{2}, 0,-1 / \sqrt{2}), \quad(0,1,0), \quad$ and $\quad(1 / 2,-1 / \sqrt{2},-1 / 2)$, respectively. ${ }^{57}$ The reflected probe light is analyzed with a polarizer set to $p$ or $s$ polarization or with no polarizer (denoted as $u$ ) and is fed to the photodetector with $2 \mathrm{MHz}$ bandwidth. The transient reflectivity change $\Delta R / R$ is recorded as a function of the delay time between the pump and probe pulse arrival at the sample surface using an optical delay line. To detect $\Delta R / R \sim 10^{-6}$, the pump light pulses are chopped with an acousto-optic modulator at $1 \mathrm{MHz}$, and the in-phase component of the photodetector output is detected with a lock-in amplifier. By the use of different wavelengths for the pump and probe light, one can avoid signal contamination by the pump light using a colored glass filter before the photodetector.

Figure 3 shows the results of the transient reflectivity measurement. A high-frequency modulation is imposed on a relatively slow-varying background that is mainly due to the temperature decay arising from thermal diffusion. Assuming a simplified single-exponential dependence, this background is subtracted to leave the signals from the acoustic wave propagation, as shown in Fig. 4. The polarization configuration is labeled on each curve as $a-b$, where $a$ is the incident light polarization $\left(p, s\right.$, and $\left.45^{\circ}\right)$ and $b$ is the detected polarization component of the reflected light $(p, s$, and $u)$.

\section{DISCUSSION}

\section{A. Qualitative analysis}

The time-domain curves in Fig. 4 show the following features: (1) a gigahertz oscillation is observed up to $400 \mathrm{ps}$, (2) three echoes are observed around 400, 700, and 800 ps, and

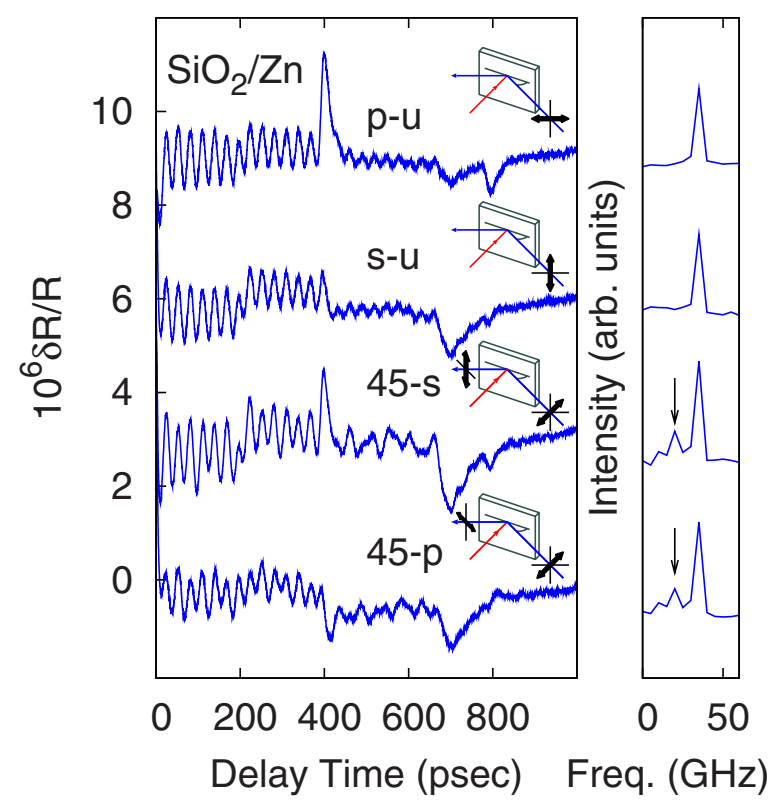

FIG. 4. (Color online) Left: Transient reflectivity change in the $\mathrm{SiO}_{2} / \mathrm{Zn}$ sample with background variation subtracted. The polarization of the incident light $\left(p, s\right.$, and $\left.45^{\circ}\right)$ and the reflected light $(p$, $s$, and $u$ ) are specified on each curve. The scales are common for all curves. Right: Fourier transforms of the transient reflectivity change up to $200 \mathrm{ps}$, revealing the shear acoustic wave components (arrows) propagating in the $\mathrm{SiO}_{2}$ layer.

(3) the oscillation and echoes lie on a background that shows steplike behavior at 200 and 400 ps.

The gigahertz oscillation is caused by the interference between the light reflected at the sample surface or at the interface and the light scattered by the strain pulse moving in the transparent $\mathrm{SiO}_{2}$ layer. The relation $v n k \cos \theta=\pi f$ applies, where $v$ is the sound speed, $n$ is the refractive index of the $\mathrm{SiO}_{2}$ layer, $k$ is the probe light wave number in vacuum, $\theta$ is the angle between the probe light propagation direction in $\mathrm{SiO}_{2}$ and the $z$ axis, and $f$ is the oscillation frequency. ${ }^{30}$ The Fourier transform of each curve up to $200 \mathrm{ps}$ is shown on the right-hand side of Fig. $4 .{ }^{58}$ For the $p$ - $u$ and $s$ - $u$ polarization configurations, a single peak is observed at $f$ $=35 \mathrm{GHz}$, corresponding to the oscillation noted above. This frequency is consistent with a sound speed $v=5.5 \mathrm{~km} \mathrm{~s}^{-1}$ (assuming $n=1.47$ ). For the $45^{\circ}-s$ and $45^{\circ}-p$ polarization configurations, an additional peak is observed at $f$ $=20 \mathrm{GHz}$, corresponding to $v=3.2 \mathrm{~km} \mathrm{~s}^{-1}$. Compared with the literature values in bulk fused silica for longitudinal and shear waves, 5.97 and $3.76 \mathrm{~km} \mathrm{~s}^{-1}$, respectively, ${ }^{31}$ one can see that the $35 \mathrm{GHz}$ signal corresponds to longitudinal waves in the $\mathrm{SiO}_{2}$ layer, whereas the $20 \mathrm{GHz}$ signal corresponds to the shear wave therein. Similarly reduced values for the sound velocity in thin silica films have been previously observed. $^{32,33}$

The fact that the shear wave is observed in the $\mathrm{SiO}_{2}$ layer only with the $45^{\circ}-s$ and $45^{\circ}-p$ configurations can be understood as a selection rule of Brillouin scattering. ${ }^{34,35,59}$ The photoelastic tensor for an isotropic medium has the form 


$$
\left(\begin{array}{cccccc}
P_{11} & P_{12} & P_{12} & 0 & 0 & 0 \\
P_{12} & P_{11} & P_{12} & 0 & 0 & 0 \\
P_{12} & P_{12} & P_{11} & 0 & 0 & 0 \\
0 & 0 & 0 & P_{44} & 0 & 0 \\
0 & 0 & 0 & 0 & P_{44} & 0 \\
0 & 0 & 0 & 0 & 0 & P_{44}
\end{array}\right),
$$

with $P_{44}=\left(P_{11}-P_{12}\right) / 2$. Since the shear wave here only has a nonvanishing $\eta_{4}$ strain component, the only modulated component in the permittivity tensor is the off-diagonal component $\varepsilon_{4}=\varepsilon_{y z}$. This has an effect on the electric field of the incident and scattered light: the sample scatters the $z$ component to the $y$ component and vice versa. In our configuration with obliquely incident probe light, $p$-polarized light is scattered into $s$-polarized light and vice versa. ${ }^{36}$ In the $p-u$ or $s-u$ configurations, however, the scattered component has an intensity proportional to $\eta_{4}^{2}$ and is too small to be detected. On the other hand, in the $45^{\circ}-p(s)$ configuration, the incident $s$ $(p)$ component is scattered to a $p(s)$ component, which interferes with the reflected $p(s)$ component to produce an intensity modulation proportional to $\eta_{4}$. It is therefore feasible to detect $\eta_{4}$ in the $45^{\circ}-p$ and $45^{\circ}-s$ configurations. Normally incident probe light does not contain a $z$ component of the electric field and therefore does not interact with the $z$-propagating shear strain in an isotropic medium.

The three echoes are mainly caused by the photoelastic effect in the $\mathrm{Zn}$ substrate. The acoustic pulses generated near the $\mathrm{SiO}_{2} / \mathrm{Zn}$ interface propagate in $\mathrm{SiO}_{2}$ layer and are then reflected at the $\mathrm{SiO}_{2}$ surface. We therefore attribute the echoes at 400 and 800 ps to the first and second returns of longitudinal-acoustic pulses to the $\mathrm{Zn}$ substrate. The polarity of the strain is flipped on reflection at the surface but stays the same on the reflection at the $\mathrm{SiO}_{2} / \mathrm{Zn}$ interface. Hence the first and second echoes have opposite polarity. The echo at 700 ps does not occur at an integer multiple of 400 ps. It can be attributed to the first return of the shear acoustic pulse to the $\mathrm{Zn}$ substrate. The ratio of arrival times (700 and 400 ps) corresponds to the inverse ratio of the sound speeds (5.5 and $3.2 \mathrm{~km} \mathrm{~s}^{-1}$ ).

The selection rule for shear strain detection in $\mathrm{Zn}$ is less restrictive compared to that in the $\mathrm{SiO}_{2}$ layer. For a $\mathrm{Zn}$ crystal with its $c$ axis inclined at $45^{\circ}$ as in the experiment here, the photoelastic tensor takes the form

$$
\left(\begin{array}{cccccc}
P_{11} & P_{12} & P_{13} & P_{14} & 0 & 0 \\
P_{21} & P_{22} & P_{23} & P_{24} & 0 & 0 \\
P_{31} & P_{32} & P_{33} & P_{34} & 0 & 0 \\
P_{41} & P_{42} & P_{43} & P_{44} & 0 & 0 \\
0 & 0 & 0 & 0 & P_{55} & P_{56} \\
0 & 0 & 0 & 0 & P_{65} & P_{66}
\end{array}\right) .
$$

In this case, the shear strain $\eta_{4}$ can modulate the diagonal components of the permittivity tensor, such as $\varepsilon_{1}$ or $\varepsilon_{2}$, as well as the off-diagonal components. In addition, the acoustic modes propagating in the $z$ direction in the $\mathrm{Zn}$ substrate are quasishear and quasilongitudinal modes containing dominant shear and longitudinal components, respectively (see
Fig. 6). For these reasons the arrival of shear acoustic pulses from the $\mathrm{SiO}_{2}$ film at the $\mathrm{Zn}$ substrate is clearly seen in all the polarization configurations used.

The steplike behavior at 200 ps and 400 ps is caused by the motion of the surface and interface that affects the variations in optical reflectivity owing to multiple optical reflections in the $\mathrm{SiO}_{2}$ layer. ${ }^{20}$

\section{B. Quantitative analysis}

For quantitative analysis, we carry out a simulation consisting of three parts: the generation, the propagation, and the optical detection of the acoustic waves. For the acoustic generation, the simplest model assumes that the absorbed pump light energy is instantaneously converted to thermal energy in the absence of diffusion processes. In this case the initial thermal stress distribution is determined by that of the absorbed pump light energy, implying that the acoustic pulse width is determined by the pump light penetration depth. However, for $\mathrm{Zn}$ at a pump wavelength of $814 \mathrm{~nm}$, the optical penetration depth is $\sim 14 \mathrm{~nm}$, which cannot explain the observed relatively broad echo at 700 ps. One should therefore consider the effects of ultrafast electron and thermal diffusion in $\mathrm{Zn} .{ }^{37}$

Ultrafast nonequilibrium and equilibrium electron diffusion (the latter being equivalent to thermal diffusion) can be described by the two-temperature model that determines the nonequilibrium energy relaxation of the electron system to the lattice system. ${ }^{38}$ These systems are assumed to be independently thermalized to temperatures $T_{e}$ and $T_{l}$, respectively. The absorbed light first transfers its energy to the electrons according to the source function $S(z, t)$, the deposited energy per unit volume per unit time. The electrons diffuse while the electron and lattice systems interact through the electron-phonon interaction, mediated by the electronphonon coupling constant $g$. The coupled electron and lattice temperatures satisfy the following equations:

$$
\begin{gathered}
C_{e} \frac{\partial T_{e}}{\partial t}=\frac{\partial}{\partial z}\left(\kappa \frac{\partial T_{e}}{\partial z}\right)-g\left(T_{e}-T_{l}\right)+S(z, t), \\
C_{l} \frac{\partial T_{l}}{\partial t}=g\left(T_{e}-T_{l}\right),
\end{gathered}
$$

where $C_{e}$ and $C_{l}$ are the specific heat per unit volume of the electron and lattice systems, respectively, and $\kappa$ is the thermal conductivity. $C_{e}$ is proportional to $T_{e}$ according to $C_{e}$ $=\gamma T_{e}$, and $\kappa$ depends on $T_{e}$ and $T_{l}$ according to $\kappa=\kappa_{0} T_{e} / T_{l}$, where $\kappa_{0}$ is the thermal conductivity when the electron and lattice systems are in equilibrium. ${ }^{39,40}$ (In metals, it is appropriate to neglect the phonon contribution to the thermal conductivity since it is small in comparison to the electronic contribution. We also neglect the thermal conductivity of the $\mathrm{SiO}_{2}$ film.) In $\mathrm{Zn}$ there is only a small anisotropy in the thermal conductivity $(\sim 5 \%)$, and so we use the polycrystalline average $\kappa_{0}=116 \mathrm{Wm}^{-1} \mathrm{~K}^{-1}$ at $300 \mathrm{~K}$ here. ${ }^{41,42}$ Equation (37) is nonlinear and only solvable numerically. We used a finite difference time-domain method with the parameters given in Table I. We approximate the source function to the form 
TABLE I. Parameters for $\mathrm{Zn}$ used in the two-temperature model. Values are taken from literature except $g$ which is obtained by fitting.

\begin{tabular}{ll}
\hline \hline Thermal conductivity $^{\mathrm{a}} \kappa_{0}\left(\mathrm{~W} \mathrm{~m}^{-1} \mathrm{~K}^{-1}\right)$ & $1.16 \times 10^{2}$ \\
Lattice specific heat $^{\mathrm{a}} C_{l}\left(\mathrm{~J} \mathrm{~m}^{-3} \mathrm{~K}^{-1}\right)$ & $2.77 \times 10^{6}$ \\
Constant for electron specific heat ${ }^{\mathrm{b}} \gamma\left(\mathrm{J} \mathrm{m}^{-3} \mathrm{~K}^{-2}\right)$ & $6.98 \times 10^{1}$ \\
Electron-phonon coupling constant $g\left(\mathrm{~W} \mathrm{~m}^{-3} \mathrm{~K}^{-1}\right)$ & $6 \times 10^{16}$ \\
\hline \hline
\end{tabular}

${ }^{\mathrm{a}}$ Reference 42.

${ }^{\mathrm{b}}$ Reference 43 .

$$
S(z, t)=S_{0} \delta(t) \exp \left\{-\left(z-z_{1}\right) / \zeta\right\} \quad \text { for } \quad z>z_{1} \text {. }
$$

The pump light optical penetration depth $\zeta$ for $\mathrm{Zn}$ is taken as $14 \mathrm{~nm}$ (see Table II). The electron-phonon coupling constant $g$ is adjusted for optimum agreement with experiment. The maximum electron and lattice temperature rises are estimated to be $\sim 300$ and $\sim 1 \mathrm{~K}$, respectively. The initial lattice temperature reaches its maximum within 1 ps after the pump pulse arrival, and then the temperature decreases slowly with a rate no greater than $4 \times 10^{-3} \mathrm{~K} \mathrm{ps}^{-1}$. Although the theory accounts for nonlinear effects, the acoustically induced reflectance changes are predicted to vary nearly linearly with pump fluence up to the fluences used. This was verified in experiment.

The sound velocity and acoustic polarization for the modes in each layer are calculated from Eq. (16). Equations (21)-(23) are then used to find the thermoelastic stress for each mode based on the lattice temperature rise $\Delta T(z, t)$ obtained with the two-temperature model. The acoustic wave propagation along both positive and negative $z$ directions is traced step by step ${ }^{21}$ in the time domain with a $\Delta t=1$ ps time step. The temporal variation of $\Delta T(z, t)$ is taken into account using Eq. (25), that is, at each time step, the temperature rise within the latest step period adds propagating and static components to the strain field. At the interface, the reflection and transmission of each mode including mode conversion is calculated using Eq. (24) (see also Appendix C).

The result is a longitudinal-acoustic pulse in the $\mathrm{SiO}_{2}$ with an initially generated frequency of $\sim 50 \mathrm{GHz}$ and a shear acoustic pulse with a initially generated frequency of $\sim 20 \mathrm{GHz}$. The effect of the much higher frequency strain generation from the electron system (dependent on $\partial T_{e} / \partial t$ ) is ignored because of the relatively strong ultrasonic attenuation at terahertz frequencies. ${ }^{37}$

Although the above procedure is sufficient for numerical calculation of the strain generation and propagation, it is also useful to make rough estimates of the acoustic pulse spatial extent using analytical expressions. According to the $\sim 700$ fs electron energy relaxation time $\left(\tau_{e} \equiv C_{e} / g\right.$ with parameters in Table I at the maximum electron temperature 600 $\mathrm{K})$, the electron and lattice temperatures become nearly equal within time $\tau_{e}$ as the electron energy becomes distributed over a depth $z_{e} \sim\left(\tau_{e} \kappa / C_{e}\right)^{1 / 2}=(\kappa / g)^{1 / 2} \sim 70 \mathrm{~nm}$ in the Zn. ${ }^{8,37}$ Nonequilibrium electron diffusion thus ceases for times greater than $\sim \tau_{e}$, when $T_{e}$ and $T_{l}$ are approximately equal. As shown in Eq. (25), the acoustic thermoelastic stress generation depends on $\partial \Delta T / \partial t$. The acoustic sources arising
TABLE II. Parameters taken from the literature for the simulation shown in Fig. 7.

\begin{tabular}{|c|c|}
\hline \multicolumn{2}{|c|}{ Mass density ${ }^{\mathrm{a}}\left(\mathrm{kg} / \mathrm{m}^{3}\right)$} \\
\hline $\mathrm{SiO}_{2}$ film & $2.203 \times 10^{3}$ \\
\hline $\mathrm{Zn}$ & $7.134 \times 10^{3}$ \\
\hline \multicolumn{2}{|c|}{ Electrical permittivity of $\mathrm{Zn}^{\mathrm{b}}$} \\
\hline \multicolumn{2}{|c|}{ Perpendicular to $c$ axis } \\
\hline$\varepsilon_{11}$ at $407 \mathrm{~nm}$ & $-8.27+2.56 i$ \\
\hline$\varepsilon_{11}$ at $814 \mathrm{~nm}$ & $-5.87+36.9 i$ \\
\hline \multicolumn{2}{|c|}{ Parallel to $c$ axis } \\
\hline$\varepsilon_{33}$ at $407 \mathrm{~nm}$ & $-7.05+1.77 i$ \\
\hline$\varepsilon_{33}$ at $814 \mathrm{~nm}$ & $-7.25+33.3 i$ \\
\hline \multicolumn{2}{|c|}{ Thermal expansion coefficient of $\mathrm{Zn}^{\mathrm{c}}(1 / \mathrm{K})$} \\
\hline$a_{11}$ & $1.3 \times 10^{-5}$ \\
\hline$a_{33}$ & $6.4 \times 10^{-5}$ \\
\hline \multicolumn{2}{|c|}{ Elastic stiffness of $\mathrm{Zn}^{\mathrm{a}}(\mathrm{GPa})$} \\
\hline$c_{11}$ & 163.7 \\
\hline$c_{12}$ & 36.4 \\
\hline$c_{13}$ & 53.0 \\
\hline$c_{33}$ & 63.5 \\
\hline$c_{44}$ & 38.8 \\
\hline \multicolumn{2}{|c|}{ Thermal conductivity of $\mathrm{Zn}^{\mathrm{b}}\left(\mathrm{Wm}^{-1} \mathrm{~K}^{-1}\right)$} \\
\hline$\kappa_{0}$ & $1.16 \times 10^{2}$ \\
\hline \multicolumn{2}{|c|}{ Lattice specific heat of $\mathrm{Zn}^{\mathrm{b}}\left(\mathrm{Jm}^{-3} \mathrm{~K}^{-1}\right)$} \\
\hline$C_{l}$ & $2.77 \times 10^{6}$ \\
\hline \multicolumn{2}{|c|}{$\begin{array}{l}\text { Constant for electron specific heat of } \mathrm{Zn}^{\mathrm{c}} \\
\left(\mathrm{Jm}^{-3} \mathrm{~K}^{-2}\right)\end{array}$} \\
\hline$\gamma$ & $6.98 \times 10^{1}$ \\
\hline
\end{tabular}

${ }^{\text {aReference }} 31$.

${ }^{\mathrm{b}}$ Reference 42 .

${ }^{\mathrm{c}}$ Reference 26.

${ }^{\mathrm{d}}$ Reference 43.

from this term for times $\lesssim \tau_{e}$ set up an initial stress within a depth $\sim z_{e}$ determined by nonequilibrium electron diffusion. Then, for times $\gtrsim \tau_{e}$, the slow varying $\Delta T$ governed by thermal diffusion will produce further stresses. In the case of zinc, the contributions for $t \lesssim \tau_{e}$ are dominant. ${ }^{60}$

The ultrasonic attenuation in the $\mathrm{SiO}_{2}$ layer is also included. For each acoustic mode, the acoustic absorption coefficient $\alpha_{\text {ac }}$ is assumed to be

$$
\alpha_{\mathrm{ac}}=b f^{2},
$$

where $b$ is a constant that we term the ultrasonic absorption constant and $f$ is the acoustic frequency. This type of frequency dependence is common to many materials at gigahertz frequencies. ${ }^{24}$ Because of this frequency dependence, the high-frequency components of the strain pulses are preferentially lost as the strain propagates. This $f^{2}$ attenuation is also included in the time-domain simulation. ${ }^{44}$ The ultrasonic attenuation in the $\mathrm{Zn}$ is negligible for the small acoustic penetration distances experimentally probed (i.e., the optical penetration depth) and is not included. 


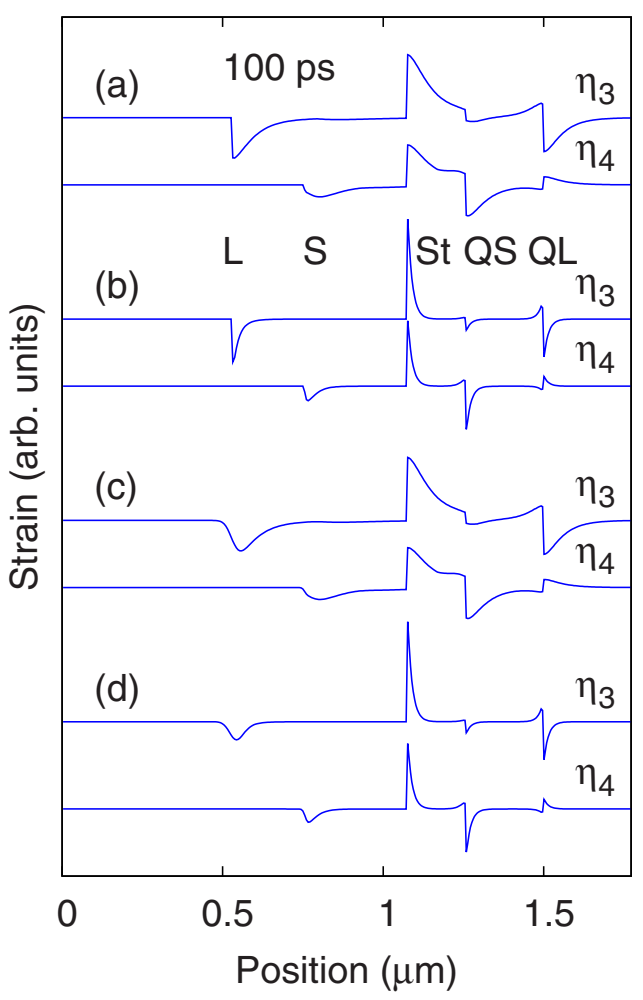

FIG. 5. (Color online) Calculated strain distribution obtained at a time $100 \mathrm{ps}$ after the arrival of a pump light pulse at the sample. (a) $\eta_{3}$ and $\eta_{4}$ according to the theory including nonequilibrium electron diffusion and ignoring ultrasonic attenuation. (b) The same quantities in the absence of diffusion processes and ignoring ultrasonic attenuation. (c) The same quantities according to the theory including nonequilibrium electron diffusion and ultrasonic attenuation. (d) The same quantities in the absence of diffusion processes but including ultrasonic attenuation. $\mathrm{L}$, longitudinal wave in $\mathrm{SiO}_{2}$; $\mathrm{S}$, shear wave in $\mathrm{SiO}_{2}$; St, static strain component; QS, quasishear wave in $\mathrm{Zn}$; and QL, quasilongitudinal wave in $\mathrm{Zn}$. The sample surface is at a position corresponding to 0 , whereas the $\mathrm{SiO}_{2} / \mathrm{Zn}$ interface is at $1.07 \mu \mathrm{m}$.

Figure 5 shows the calculated strain distribution at $100 \mathrm{ps}$ after the pump light pulse arrival. The static $(\mathrm{St})$ component corresponds to $\boldsymbol{\eta}_{B}$ and does not propagate. In the $\mathrm{SiO}_{2}$ layer pure longitudinal (L) and shear (S) pulses can propagate, whereas in Zn quasilongitudinal (QL) and quasishear (QS) pulses can propagate with corresponding velocities $v_{\mathrm{QL}}$ $=4.2 \mathrm{~km} \mathrm{~s}^{-1}$ and $v_{\mathrm{OS}}=1.8 \mathrm{~km} \mathrm{~s}^{-1}$, respectively.

First ignoring the effects of ultrasonic attenuation, we show in Fig. 5(a) the calculated strain in the presence of nonequilibrium electron diffusion, and Fig. 5(b) in the absence of diffusion processes [equivalent to the case of $\kappa=0$ in Eq. (37)]. In Fig. 5(b) the static strain component St is defined to correspond to the pump light absorption profile. In accordance with the significant electron diffusion depth $z_{e}$, the generated acoustic pulses are spatially broadened by a factor of $\sim 5$ compared to those in the absence of diffusion processes. The rounded shape of the shear pulses in $\mathrm{SiO}_{2}$ compared to that of the longitudinal pulses has been explained by Pezeril et al. ${ }^{17,61}$ as being caused by the separation of the shear strain into two components of opposite sign at $t=0$ associated with the $\mathrm{QL}$ and QS modes. The nonequi-

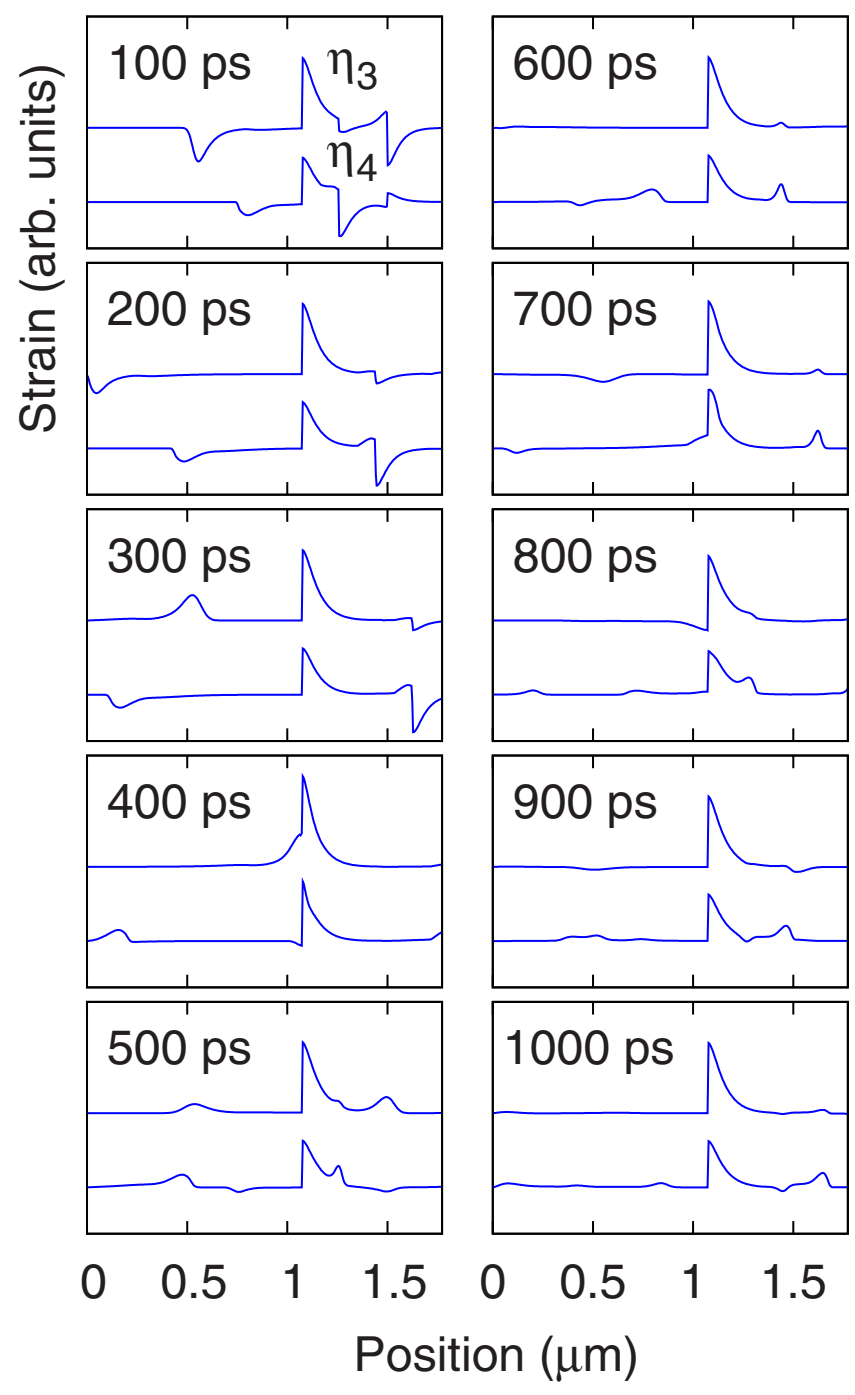

FIG. 6. (Color online) Snapshots of the strain fields $\eta_{3}$ and $\eta_{4}$ at different times after the pump light pulse arrival.

librium electron diffusion theory predicts that the spatial extent of the generated longitudinal pulses in the $\mathrm{SiO}_{2}$ is $\sim z_{e} v_{l} / v_{\mathrm{QL}} \sim 90 \mathrm{~nm}$, whereas that of the shear pulses is $\sim z_{e} v_{t} / v_{\mathrm{QS}} \sim 120 \mathrm{~nm}$, in reasonable agreement with Fig. $5(\mathrm{a})$.

Now including the effects of ultrasonic attenuation, we show in Figs. 5(c) and 5(d) the calculated strain in the presence and absence of nonequilibrium electron diffusion, respectively. The effect of the frequency dependent ultrasonic attenuation in the $\mathrm{SiO}_{2}$ layer is to slightly reduce the frequency of the acoustic pulses.

Figure 6 shows the spatiotemporal evolution of the strain components $\eta_{3}$ (longitudinal) and $\eta_{4}$ (shear), which are used in the calculation of the optical reflectivity change. The component $\eta_{5}$ is zero owing to the symmetry of the system. The $\mathrm{SiO}_{2}$ longitudinal pulse flips its polarity at the surface but not at the $\mathrm{SiO}_{2} / \mathrm{Zn}$ interface.

The electric field of the reflected probe light is calculated using the procedure given in Sec. II D, 


$$
\begin{aligned}
\boldsymbol{E}(z, t)= & \boldsymbol{E}_{0}(z)+k^{2} \int_{0}^{\infty} \boldsymbol{G}\left(z, z^{\prime}\right) \Delta \boldsymbol{\varepsilon}^{(\mathrm{pe})}\left(z^{\prime}, t\right) \boldsymbol{E}_{0}\left(z^{\prime}\right) d z^{\prime} \\
& +k^{2} \int_{0}^{\infty} \boldsymbol{G}\left(z, z^{\prime}\right) \Delta \boldsymbol{\varepsilon}^{(\mathrm{rt})}\left(z^{\prime}, t\right) \boldsymbol{E}_{0}\left(z^{\prime}\right) d z^{\prime}+\sum_{n=0}^{1} k^{2} u_{z}\left(z_{n}, t\right) \\
& \times \boldsymbol{G}\left(z, z_{n}+0\right)\left[\boldsymbol{\varepsilon}^{(n)}-\boldsymbol{\varepsilon}^{(n+1)}\right] \boldsymbol{E}_{0}\left(z_{n}-0\right) .
\end{aligned}
$$

The first, second, and third terms are caused by the photoelastic effect, by local rotations, and by surface or interface displacements, respectively.

The polarization of the incident light determines the form of $\boldsymbol{E}_{0}$. With the procedure described in Appendix A, we calculate the amplitude coefficient of reflectance $r_{j}$ for the polarization configuration $j$ out one of the following, $p-p, s-s$, $p-s$, and $s-p$, which each refers to the incident and reflected light polarizations, respectively. The polarization vectors are chosen as $(1 / \sqrt{2}, 0,-1 / \sqrt{2})$ for $p$ incident, $(0,1,0)$ for $s$ incident, $(1 / \sqrt{2}, 0,1 / \sqrt{2})$ for $p$ reflected, and $(0,1,0)$ for $s$ reflected. Then we calculate the modulation in amplitude reflectance $\delta r_{j}$ for the polarization configuration $j$ using Eq. (39). The intensity reflectivity change $\delta R_{j} / R_{j}$ for polarization configuration $j$ is given by

$$
\begin{gathered}
\frac{\delta R_{p-u}}{R_{p-u}}=2 \operatorname{Re} \frac{r_{p-p} \delta r_{p-p}^{*}+r_{p-s}^{*} \delta r_{p-s}}{r_{p-p} r_{p-p}^{*}+r_{p-s} r_{p-s}^{*}}, \\
\frac{\delta R_{s-u}}{R_{s-u}}=2 \operatorname{Re} \frac{r_{s-s} \delta r_{s-s}^{*}+r_{s-p}^{*} \delta r_{s-p}}{r_{s-s} r_{s-s}^{*}+r_{s-p} r_{s-p}^{*}}, \\
\frac{\delta R_{45-s}}{R_{45-u}}=2 \operatorname{Re} \frac{\delta r_{s-s}-\delta r_{p-s}}{r_{s-s}-r_{p-s}}, \\
\frac{\delta R_{45-p}}{R_{45-p}}=2 \operatorname{Re} \frac{\delta r_{p-p}-\delta r_{s-p}}{r_{p-p}-r_{s-p}} .
\end{gathered}
$$

These can be directly compared with the experimental results. ${ }^{62}$

Each expression in Eq. (40) consists of two types of terms, one linear in the photoelastic constants and one independent of them (related to the surface and interface displacements and to the local rotation),

$$
\frac{\delta R(t)}{R}=\sum_{n=1}^{2} P_{I J}^{(n)} F_{n I J}(t)+F_{0}(t) .
$$

The first term corresponds to the photoelastic contribution. It consists of the sum of contributions from the $n$th layer $(n$ =1,2). Each contribution $P_{I J}^{(n)} F_{n I J}(t)$ is linear in the photoelastic tensor component $P_{I J}^{(n)}$, and the coefficient $F_{n I J}(t)$ is obtained from Eqs. (39) and (40) by setting $P_{I J}^{(n)}$ to unity and all other $P$ 's to zero. $F_{0}(t)$ corresponds to the interface and rotation contributions. The form of Eq. (41) is convenient for the automatic determination of each independent photoelastic tensor component using standard least-squares fitting (involving a linear regression method) to the complete experimental data set. Tensor rotation rules, ${ }^{26}$ which determine the photoelastic constants of Eq. (27), can be incorporated into $F_{n I J}$ in Eq. (41) for convenience so that we directly obtain

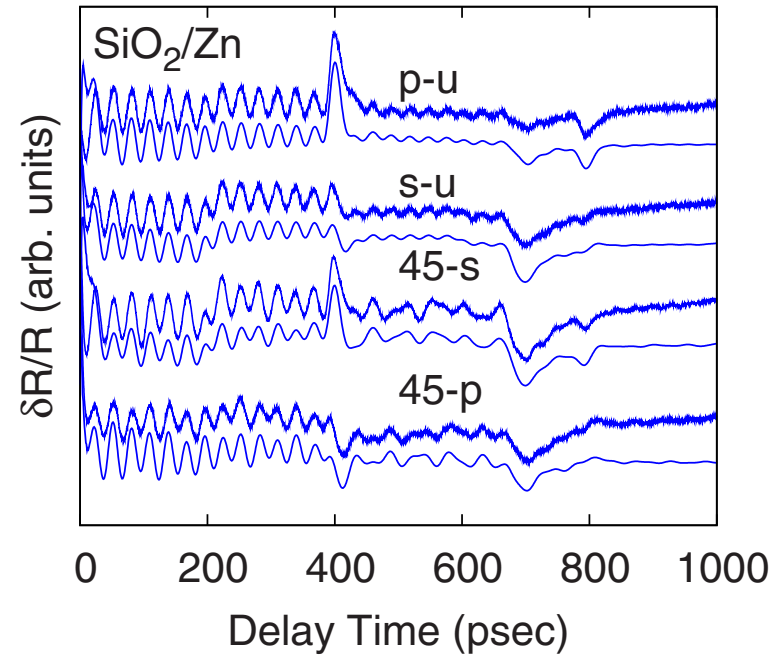

FIG. 7. (Color online) Each pair of curves correspond to experiment (upper) and fits (lower) for the transient reflectivity change in the $\mathrm{SiO}_{2} / \mathrm{Zn}$ sample. The polarization of the incident light $(p, s$, and $\left.45^{\circ}\right)$ and the reflected light $(p, s$, and $u)$ are specified on each curve. Scales are common for all curves, which are shifted vertically for clarity. All fits are obtained with common fitting parameters.

$P_{I J}^{(n)}$ 's defined for any convenient crystal orientation. The reflectivity change caused by the photoelastic effect is proportional to the photoelastic constants, whereas that caused by the surface and/or interface displacement or local rotation is independent of them. The proper inclusion of the effects of multiple optical scattering results in a nonzero value of $F_{0}(t)$ in Eq. (41) and thus allows the determination of the absolute value of the photoelastic constants..$^{20,21,63}$

Figure 7 shows a comparison of the experimental data with the optimal fits. The parameters taken from the literature are shown in Table II, whereas the fitted parameters are shown in Table III. We obtain good agreement between the experiment and the fits. The tensor components $\varepsilon_{i j}, \alpha_{i j}, c_{I J}$, and $P_{I J}$ are given for the conventional crystalline orientation.

The photoelastic constants for the $\mathrm{SiO}_{2}$ film can be compared with the literature values, ${ }^{45} P_{11}=-0.57$ and $P_{12}=$ $-1.26 .{ }^{64}$ Although the fitted value of $P_{12}$ is close to the literature value, $P_{11}$ is not. This might be due to a decrease in the generated shear strain amplitude due to degradation of the $\mathrm{Zn}$ surface. The $\mathrm{Zn}$ photoelastic constants obtained here are also strongly dependent on the assumed spatiotemporal form and the relative amplitudes of the strain field components (that are affected by the Zn surface condition). We do not know of any other measurements of $P_{I J}$ in $\mathrm{Zn}$ at our optical probe wavelength of $407 \mathrm{~nm}$. Pezeril et al. ${ }^{17}$ determined a different set at $800 \mathrm{~nm}$. Their values represent ratios with respect to a single tensor component rather than absolute values. For a precise determination of the single-crystal photoelastic constants, one should evaluate the shear strain amplitude independently and precisely for a defect-free sample. The use of a variety of optical incidence angles for the probe light would be an advantage. Our method is capable of determining absolute values of photoelastic constants, thanks to the presence of the $\mathrm{SiO}_{2}$ top layer (provided that the correct strain distribution is known). The presence of 
TABLE III. Parameters determined by the simulation shown in Fig. 7. The refractive index and photoelastic constants correspond to the optical probe wavelength of $407 \mathrm{~nm}$.

\begin{tabular}{lc} 
Sound velocity in $\mathrm{SiO}_{2}$ film & \\
Longitudinal $v_{l}(\mathrm{~m} / \mathrm{s})$ & $5.46 \times 10^{3}$ \\
Shear $v_{t}(\mathrm{~m} / \mathrm{s})$ & $3.22 \times 10^{3}$ \\
Refractive index of $\mathrm{SiO}_{2}$ film & 1.47 \\
Thickness of $\mathrm{SiO}_{2}$ film $(\mathrm{nm})$ & $1.07 \times 10^{3}$ \\
Ultrasonic absorption constant in the $\mathrm{SiO}_{2}$ layer & \\
Longitudinal $b_{l}\left(\mathrm{~m}^{-1} \mathrm{GHz}^{-2}\right)$ & $4.3 \times 10^{2}$ \\
Shear $b_{t}\left(\mathrm{~m}^{-1} \mathrm{GHz}^{-2}\right)$ & $2.0 \times 10^{2}$ \\
$\mathrm{Photoelastic} \mathrm{constants}_{\mathrm{SiO}}$ film, $P_{11}$ & \\
$\mathrm{SiO}_{2}$ film, $P_{12}$ & -1.1 \\
$\mathrm{Zn}, P_{11}$ & -1.5 \\
$\mathrm{Zn}, P_{12}$ & $30+60 i$ \\
$\mathrm{Zn}, P_{13}$ & $9-40 i$ \\
$\mathrm{Zn}, P_{31}$ & $27-38 i$ \\
$\mathrm{Zn}, P_{33}$ & $100-200 i$ \\
$\mathrm{Zn}, P_{44}$ & $200+300 i$ \\
$\mathrm{Electron}-$ phonon coupling constant of $\mathrm{Zn}$ & $120+150 i$ \\
$g\left(W \mathrm{~K}^{-3} \mathrm{~K}^{-1}\right)$ & \\
\hline \hline
\end{tabular}

the oscillating component and the underlying steps in the signals from the transparent layer are an advantage for the determination of $P_{I J}$.

Figure 8 shows the separate photoelastic, surface and interface displacements, and local rotation contributions to the transient reflectivity for the four experimental configurations. The oscillations arise from the photoelastic contribution in the $\mathrm{SiO}_{2}$ layer. The echoes (corresponding to the acoustic pulse arrivals at the $\mathrm{Zn}$ interface) are also mainly determined by this contribution. The steplike variation arises from the surface or interface displacements induced by the acoustic propagation near these discontinuities. The local rotation contribution has a shape that mimics the acoustic strain variation in the region of the interface. Its amplitude is much smaller than the other contributions.

The parameters other than the photoelastic constants in Table III are chosen by iterative search using a least-squares method. (The photoelastic constants are determined by a noniterative least-squares method as discussed before.) This fitting procedure is complicated by the interrelation between parameters. A brief discussion of this is given here.

The sound velocity, refractive index, and thickness of the $\mathrm{SiO}_{2}$ layer are mutually related. The arrival time of the first echoes for longitudinal and shear waves traveling in the $\mathrm{SiO}_{2}$ layer (400 and $700 \mathrm{ps)}$ correspond to the traversed thickness divided by the relevant sound velocity. The frequency of the oscillations caused by the photoelastic effect in the $\mathrm{SiO}_{2}$ layer $(20$ and $35 \mathrm{GHz}$ ) is determined by the sound velocity and refractive index. As mentioned previously, the derived sound velocities are close to literature values. The refractive index of the sputtered $\mathrm{SiO}_{2}$ layer is also close to the literature value at our probe wavelength $(n=1.46$ for bulk fused silica, ${ }^{42}$ and $n=1.51$ for sputtered films of silica $\left.{ }^{46}\right)$. We esti-

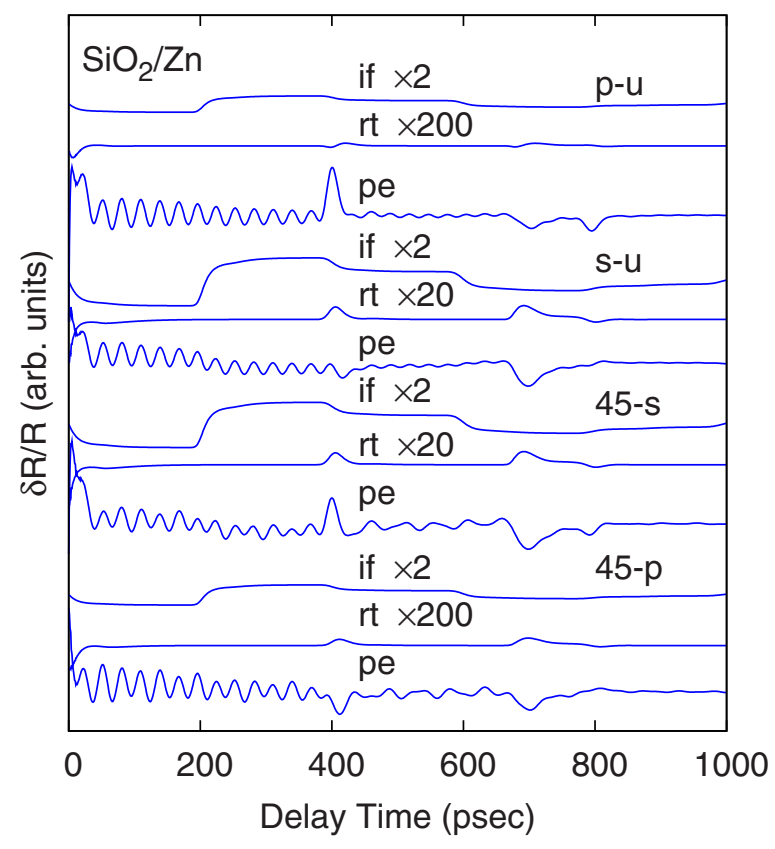

FIG. 8. (Color online) The contributions from photoelasticity (pe), surface and interface displacements (if), and local rotations (rt) to the simulated transient reflectivity shown in Fig. 7.

mate that the error in our sound velocities, thicknesses, and refractive index here is no more than $1 \%$.

The ultrasonic absorption constant $b$ for longitudinal and shear waves in the $\mathrm{SiO}_{2}$ layer has a strong effect on the echo shape at 400 and 700 ps (corresponding to the longitudinal and shear wave arrival at the $\mathrm{Zn}$ ). A larger value of $b$ tends to give a more rounded echo. The ultrasonic absorption coefficients in bulk vitreous silica obtained by Brillouin scattering ${ }^{47}$ are $7200 \mathrm{~dB} \mathrm{~cm}^{-1}$ at $35 \mathrm{GHz}$ for longitudinal waves and $3700 \mathrm{~dB} \mathrm{~cm}^{-1}$ at $16 \mathrm{GHz}$ for shear waves, which correspond to amplitude absorption coefficients $\alpha_{\mathrm{ac}}=8.3$ $\times 10^{4}$ and $4.3 \times 10^{4} \mathrm{~m}^{-1}$, respectively. The latter gives $b$ $=170 \mathrm{~m}^{-1} \mathrm{GHz}^{-2}$ for shear waves, which is very close to the value in Table III, whereas the former gives $b$ $=70 \mathrm{~m}^{-1} \mathrm{GHz}^{-2}$ for longitudinal waves, which is significantly smaller than the value in Table III. Picosecond acoustics measurements ${ }^{30}$ for bulk vitreous silica for longitudinal waves at $30 \mathrm{GHz}$ give a similarly reduced value $b$ $=110 \mathrm{~m}^{-1} \mathrm{GHz}^{-2}$. The reason for the difference between these bulk and thin-film values is not clear. We estimate that the error in our determination of $b$ is no more than $10 \%$.

The electron-phonon coupling constant $g$ for $\mathrm{Zn}$ determines the duration of the generated strain pulses as described in Sec. IV B. The predicted duration of the shear wave echo at $700 \mathrm{ps}$ (corresponding to the shear wave arrival at the $\mathrm{Zn}$ ) is $\sim z_{e} / v_{Q S}=40 \mathrm{ps}$. The duration of the longitudinal wave echo at $400 \mathrm{ps}$ is $\sim z_{e} / v_{Q L}=20 \mathrm{ps}$. (These estimates are a little smaller than the experimental durations because of the effects of the ultrasonic absorption, and one should bear in mind that they are only order-of-magnitude estimates.) For the case of no ultrafast diffusion $(g=\infty$ or $\kappa=0)$, the echoes become unreasonably short (as is clear from Fig. 5). The fitted value of $g=6 \times 10^{16} \mathrm{~W} \mathrm{~m}^{-3} \mathrm{~K}^{-1}$ agrees very well with the literature value ${ }^{48,49} g=6.4 \times 10^{16} \mathrm{~W} \mathrm{~m}^{-3} \mathrm{~K}^{-1}$. We esti- 
mate that the error in our determination of $g$ is no more than $20 \%$.

The photoelastic constants of the $\mathrm{SiO}_{2}$ layer and the $\mathrm{Zn}$ crystal affect the sign and magnitude of the oscillatory and echo signals. Owing to the large number of photoelastic constants for $\mathrm{Zn}$, some of our values are not precisely determined. The values of $P_{12}$ for the $\mathrm{SiO}_{2}$ layer are relatively well determined to within $20 \%$. However, the only photoelastic constants that are known within $30 \%$ are $P_{11}$ for $\mathrm{SiO}_{2}$, $P_{13}$ for $\mathrm{Zn}$, and $P_{44}$ for $\mathrm{Zn}$. As for the other components for $\mathrm{Zn}, P_{12}$ and $P_{33}$ are known to within $100 \%$, and $P_{11}$ and $P_{31}$ within $300 \%$. These poor accuracies indicate that the information from the observed signals at our single angle of probe incidence is not sufficient for a more precise determination.

\section{SUMMARY}

We have described in detail experiments at oblique optical incidence using ultrashort optical pulses to generate and detect picosecond acoustic pulses in a thin layer of silica on a crystalline zinc substrate. The results are analyzed with a light-scattering theory for arbitrary optical incidence angle and polarization in thin multilayers of isotropic or anisotropic media containing an inhomogeneous perturbation in the permittivity tensor in the depth direction. The theory is applied to a sample of $\mathrm{SiO}_{2}$ on an angle-cut $\mathrm{Zn}$ substrate in which both longitudinal and shear acoustic waves can be optically generated. Fitting the theory to experiment allowed us to derive acoustic and optical constants to varying degrees of accuracy.

The method we have presented can be applied to a variety of samples. We and other investigators ${ }^{17}$ have barely scraped the surface of this interesting mixture of anisotropic optics and acoustics. Partially transparent anisotropic layers, including ferroelectric or piezoelectric multilayers, should prove to be extremely interesting for similar future studies. We also look forward to a range of experiments at different angles of optical incidence and different wavelengths to better characterize the optoacoustic response of not only metals and insulators but also of semiconductor multilayers.

\section{ACKNOWLEDGMENTS}

We are grateful for discussions with Madeleine Msall, Thomas Pezeril, and Thomas Dehoux. This work is partially supported by a Grant-in-Aid for Scientific Research from the Ministry of Education, Science, Sports, and Culture, Japan and by the 21st Century Center of Excellence (COE) program entitled Topological Science and Technology. Part of this work was supported by the Japan Society for the Promotion of Science (JSPS).

\section{APPENDIX A: TRANSFER MATRIX METHOD}

Light propagation in a piecewise homogeneous medium can be described using the transfer-matrix method. ${ }^{50-52}$ Since it is also important to derive the Green's function, we describe in detail how to obtain solutions of the electromagnetic wave equation [Eq. (8)] in such a medium. We first discuss Eq. (4) in the homogeneous case, for which $\varepsilon$ takes a constant value. The solution can be expressed as a plane wave in the form

$$
\boldsymbol{E}(z)=\boldsymbol{e} \exp \left\{i k_{z} z\right\}
$$

where $\boldsymbol{e}$ is a constant polarization vector. Substituting Eq. (A1) into Eq. (4), we get

$$
\left\{\left(\begin{array}{ccc}
-k_{z}^{2} & 0 & k_{x} k_{z} \\
0 & -k_{z}^{2}-k_{x}^{2} & 0 \\
k_{x} k_{z} & 0 & -k_{x}^{2}
\end{array}\right)+k^{2} \boldsymbol{\varepsilon}\right\} \boldsymbol{e}=0 .
$$

The secular equation

$$
\left|\left(\begin{array}{ccc}
-k_{z}^{2} & 0 & k_{x} k_{z} \\
0 & -k_{z}^{2}-k_{x}^{2} & 0 \\
k_{x} k_{z} & 0 & -k_{x}^{2}
\end{array}\right)+k^{2} \varepsilon\right|=0
$$

gives four possible values of $k_{z}$ for a given set of $k_{x}$ and $k$ (or $\omega)$. Two out of the four propagate (or decrease) in the $+z$ direction and the other two propagate (or decrease) in the $-z$ direction. We denote the former two as $k_{1}$ and $k_{2}$, and the latter two as $k_{3}$ and $k_{4}$. By substituting $k_{\mu}(\mu=1, \ldots, 4)$ into Eq. (A2), the corresponding optical polarization vector $\boldsymbol{e}_{\mu}$ is obtained.

We now consider the solution for a medium with piecewise homogeneity, as shown in Fig. 1 . Within the $n$th layer, the solution is expressed as

$$
\boldsymbol{E}(z)=a_{\mu}^{(n)} \boldsymbol{e}_{\mu}^{(n)} \exp \left\{i k_{\mu}^{(n)} \zeta^{(n)}\right\},
$$

where $a_{\mu}$ 's are the amplitudes of each mode and $\zeta^{(n)}$ is defined as

$$
\zeta^{(n)} \equiv \begin{cases}z-z_{n-1} & \text { for } n \geq 1 \\ z & \text { for } n=0 .\end{cases}
$$

Superscripts $(n)$ are used to specify the layer. The summation is taken for $\mu=1, \ldots, 4$.

At each interface, we must have continuity in the tangential components of the electric field $\boldsymbol{E}$ and magnetic field $\boldsymbol{H}$ and in the normal components of electric displacement $\boldsymbol{D}$ and magnetic-flux density $\boldsymbol{B}$. Taking the magnetic permeability as unity throughout the medium, this leads to four independent conditions for the continuity of $E_{x}, E_{y}, B_{x}$, and $B_{y}$. These four conditions at the interface between the $n$th and $(n$ $+1)$ th layers are conveniently expressed in a matrix form,

$$
\boldsymbol{A}_{n} \boldsymbol{P}_{n} \boldsymbol{a}_{n}=\boldsymbol{A}_{n+1} \boldsymbol{a}_{n+1} \text {. }
$$

The components of the $4 \times 4$ matrices $\boldsymbol{A}_{n}$ and $\boldsymbol{P}_{n}$ as well as the column vector $\boldsymbol{a}_{n}$ are defined as

$$
\begin{gathered}
{\left[\boldsymbol{A}_{n}\right]_{1 \mu} \equiv e_{x \mu}^{(n)},} \\
{\left[\boldsymbol{A}_{n}\right]_{2 \mu} \equiv e_{y \mu}^{(n)},} \\
{\left[\boldsymbol{A}_{n}\right]_{3 \mu} \equiv k_{\mu}^{(n)} e_{x \mu}^{(n)}-k_{x} e_{z \mu}^{(n)},} \\
{\left[\boldsymbol{A}_{n}\right]_{4 \mu} \equiv k_{\mu}^{(n)} e_{y \mu}^{(n)},} \\
{\left[\boldsymbol{P}_{n}\right]_{\mu \nu} \equiv \exp _{i}\left\{k_{\mu}^{(n)} d_{n}\right\} \delta_{\mu \nu} \text { for } n \neq 0,}
\end{gathered}
$$




$$
\begin{gathered}
{\left[\boldsymbol{P}_{0}\right]_{\mu \nu} \equiv \delta_{\mu \nu},} \\
{\left[\boldsymbol{a}_{n}\right]_{\mu} \equiv a_{\mu}^{(n)},}
\end{gathered}
$$

where $\delta_{\mu \nu}$ is Kronecker's delta and $e_{j \mu}$ denotes the $j$ th component of vector $\boldsymbol{e}_{\mu}$. No summation on $\mu$ is required here. For a medium with $N$ layers on a substrate, there are $4 N+8$ amplitude components and $4 N+4$ boundary conditions. If we specify four arbitrary amplitude components out of $4 N+8$, the remaining amplitude components are uniquely determined by Eq. (A6). For example, the reflectivity and transmittivity of the medium can be obtained by setting $a_{3}^{(N+1)}$ $=a_{4}^{(N+1)}=0$ and $a_{1}^{(0)}=a, a_{2}^{(0)}=b$ for the incident light polarization. We use the notation

$$
\boldsymbol{E}\left(z ; k_{x} ; a_{1}^{(0)}=a, a_{2}^{(0)}=b, a_{3}^{(N+1)}=a_{4}^{(N+1)}=0\right)
$$

to express this particular solution. The lateral component of the wave vector $\left(k_{x}\right)$ is explicitly shown.

\section{APPENDIX B: DERIVATION OF THE GREEN'S FUNCTION}

Here we solve Eq. (9) for given $k_{x}$ and $\omega$. We use the notation $\boldsymbol{G}\left(z, z^{\prime} ; k_{x}\right)$ to specify $k_{x}$ explicitly. As shown in Eq. (11), we need $\boldsymbol{G}\left(z, z^{\prime}\right)$ for $z^{\prime} \in(-\infty, \infty)$ with $z$ at the observation point. On the other hand, as shown later, it is easier to solve Eq. (9) for $z \in(-\infty, \infty)$ rather than for $z^{\prime} \in(-\infty, \infty)$. This turns out not to be a problem because of the reciprocity of the Green's function,

$$
\begin{aligned}
& \int_{-\infty}^{\infty}{ }^{t} \boldsymbol{G}\left(z, z^{\prime} ;-k_{x}\right)\left[\left\{\boldsymbol{L}\left(k_{x}\right)+k^{2} \boldsymbol{\varepsilon}_{h}(z)\right\} \boldsymbol{G}\left(z, z^{\prime \prime} ; k_{x}\right)\right] d z \\
& \quad-\int_{-\infty}^{\infty}{ }^{t}\left[\left\{\boldsymbol{L}\left(-k_{x}\right)+k^{2} \boldsymbol{\varepsilon}_{h}(z)\right\} \boldsymbol{G}\left(z, z^{\prime} ;-k_{x}\right)\right] \boldsymbol{G}\left(z, z^{\prime \prime} ; k_{x}\right) d z \\
& =\boldsymbol{G}\left(z^{\prime}, z^{\prime \prime} ; k_{x}\right)-{ }^{t} \boldsymbol{G}\left(z^{\prime \prime}, z^{\prime} ;-k_{x}\right)=0
\end{aligned}
$$

The calculation starts with Eq. (9). This is evaluated by partial integration, making use of the symmetry $\varepsilon_{h}={ }^{t} \varepsilon_{h}$ and an infinitesimal absorption in $\boldsymbol{G}\left(z, z^{\prime} ; \pm k_{x}\right)$ that assures that $\boldsymbol{G}$ vanishes at $z \rightarrow \pm \infty$. One therefore needs to solve

$$
\left\{\boldsymbol{L}\left(-k_{x}\right)+k^{2} \boldsymbol{\varepsilon}_{h}(z)\right\} \boldsymbol{G}\left(z, z^{\prime} ;-k_{x}\right)=-\delta\left(z-z^{\prime}\right) \boldsymbol{I}
$$

for $z \in(-\infty, \infty)$. Equation (B1) is then used to obtain $\boldsymbol{G}\left(z, z^{\prime} ; k_{x}\right)$ for $z^{\prime} \in(-\infty, \infty)$.

Equation (B2) is equivalent to

$$
\left\{\boldsymbol{L}\left(-k_{x}\right)+k^{2} \boldsymbol{\varepsilon}_{h}(z)\right\} \boldsymbol{E}_{0}\left(z ;-k_{x}\right)=0
$$

at $z \neq z^{\prime}$. The general solution for Eq. (B3) is readily obtained by the procedure described in Appendix A involving the substitution $k_{x} \rightarrow-k_{x}$. The $\delta$ function on the right-hand side of Eq. (B2) arises from the singularity in $\boldsymbol{G}\left(z, z^{\prime} ;-k_{x}\right)$ at $z=z^{\prime}$, which may be a delta function, a discontinuity, or a discontinuity in the first $z$ derivative. ${ }^{53}$ Therefore the possible form of $\boldsymbol{G}\left(z, z^{\prime} ;-k_{x}\right)$ is given as

$$
\boldsymbol{G}_{j}\left(z, z^{\prime} ;-k_{x}\right)=\boldsymbol{G}_{j}^{\prime}\left(z, z^{\prime}\right)+\boldsymbol{\Delta}_{j}\left(z^{\prime}\right) \delta\left(z-z^{\prime}\right),
$$

$$
\boldsymbol{G}_{j}^{\prime}\left(z, z^{\prime}\right)=\left\{\begin{array}{l}
g_{1 j}\left(z^{\prime}\right) \boldsymbol{E}_{1}(z)+g_{2 j}\left(z^{\prime}\right) \boldsymbol{E}_{2}(z) \text { for } z<z^{\prime} \\
g_{3 j}\left(z^{\prime}\right) \boldsymbol{E}_{3}(z)+g_{4 j}\left(z^{\prime}\right) \boldsymbol{E}_{4}(z) \text { for } z>z^{\prime},
\end{array}\right.
$$

where $\boldsymbol{G}_{j}$ is the $j$ th column of $\boldsymbol{G}$. The quantities $\boldsymbol{\Delta}_{j}$ and $g_{l j}$ depend only on $z^{\prime}$ and are determined below. The quantities $\boldsymbol{E}_{l}(l=1, \ldots, 4)$ are the particular solutions of Eq. (B3) in the form

$$
\begin{gathered}
\boldsymbol{E}_{1}(z) \equiv \boldsymbol{E}_{0}\left(z ;-k_{x} ; b_{1}^{(0)}=b_{2}^{(0)}=b_{4}^{(0)}=0, b_{3}^{(0)}=1\right) \\
\equiv b_{1 \mu}^{(n)} \boldsymbol{e}_{\mu}^{(n)} \exp \left\{i k_{\mu}^{(n)} \zeta^{(n)}\right\}, \\
\boldsymbol{E}_{2}(z) \equiv \boldsymbol{E}_{0}\left(z ;-k_{x} ; b_{1}^{(0)}=b_{2}^{(0)}=b_{3}^{(0)}=0, b_{4}^{(0)}=1\right) \\
\equiv b_{2 \mu}^{(n)} \boldsymbol{e}_{\mu}^{(n)} \exp \left\{i k_{\mu}^{(n)} \zeta^{(n)}\right\}, \\
\boldsymbol{E}_{3}(z) \equiv \boldsymbol{E}_{0}\left(z ;-k_{x} ; b_{1}^{(N+1)}=1, b_{2}^{(N+1)}=b_{3}^{(N+1)}=b_{4}^{(N+1)}=0\right) \\
\equiv b_{3 \mu}^{(n)} \boldsymbol{e}_{\mu}^{(n)} \exp \left\{i k_{\mu}^{(n)} \zeta(n)\right\}, \\
\boldsymbol{E}_{4}(z) \equiv \boldsymbol{E}_{0}\left(z ;-k_{x} ; b_{2}^{(N+1)}=1, b_{1}^{(N+1)}=b_{3}^{(N+1)}=b_{4}^{(N+1)}=0\right) \\
\equiv b_{4 \mu}^{(n)} \boldsymbol{e}_{\mu}^{(n)} \exp \left\{i k_{\mu}^{(n)} \zeta^{(n)}\right\},
\end{gathered}
$$

where $n$ indicates the layer in which $z$ is involved, and $b_{l \mu}^{(n)}$ is the amplitude of mode $\mu$ in the $n$th layer. This amplitude is determined for the boundary conditions stated in the parentheses of $\boldsymbol{E}_{0}$ using the procedure given in Appendix A. The modes $\mu=1,2$ correspond to $\operatorname{Re}\left(k_{\mu}\right)>0$ or $\operatorname{Im}\left(k_{\mu}\right)>0$, and the modes $\mu=3,4$ correspond to $\operatorname{Re}\left(k_{\mu}\right)<0$ or $\operatorname{Im}\left(k_{\mu}\right)<0$. Note that $k_{\mu}$ and $e_{\mu}$ in this appendix are for $-k_{x}$, and slightly different from those for $k_{x}$ in Appendix A. By denoting the latter as $k_{\mu}^{\prime}$ and $e_{\mu}^{\prime}$, we can write $k_{1}=-k_{3}^{\prime}, k_{2}=-k_{4}^{\prime}, k_{3}=-k_{1}^{\prime}$, $k_{4}=-k_{2}^{\prime}, e_{1}=e_{3}^{\prime}, e_{2}=e_{4}^{\prime}, e_{3}=e_{1}^{\prime}, e_{4}=e_{2}^{\prime}$.

It is convenient to express $\boldsymbol{L}\left(-k_{x}\right)$ as

$$
\boldsymbol{L}\left(-k_{x}\right) \equiv \boldsymbol{L}_{0}+\boldsymbol{L}_{1} \frac{\partial}{\partial z}+\boldsymbol{L}_{2} \frac{\partial^{2}}{\partial z^{2}},
$$

where

$$
\begin{gathered}
\boldsymbol{L}_{0} \equiv-k_{x}^{2}\left(\begin{array}{ccc}
0 & 0 & 0 \\
0 & 1 & 0 \\
0 & 0 & 1
\end{array}\right), \boldsymbol{L}_{1} \equiv i k_{x}\left(\begin{array}{lll}
0 & 0 & 1 \\
0 & 0 & 0 \\
1 & 0 & 0
\end{array}\right) \\
\boldsymbol{L}_{2} \equiv\left(\begin{array}{lll}
1 & 0 & 0 \\
0 & 1 & 0 \\
0 & 0 & 0
\end{array}\right)
\end{gathered}
$$

With this notation, Eq. (B2) is rewritten as

$$
\begin{aligned}
{\left[\boldsymbol{L}_{0}\right.} & \left.+\boldsymbol{L}_{1} \frac{\partial}{\partial z}+\boldsymbol{L}_{2} \frac{\partial^{2}}{\partial z^{2}}+k^{2} \boldsymbol{\varepsilon}_{h}(z)\right] \boldsymbol{G}_{j}\left(z, z^{\prime} ;-k_{x}\right) \\
& =\left[\left(\boldsymbol{L}_{0}+k^{2} \boldsymbol{\varepsilon}_{h}\left(z^{\prime}\right)\right) \boldsymbol{\Delta}_{j}+\boldsymbol{L}_{1}\left\{\boldsymbol{G}_{j}^{\prime}\left(z^{\prime}+0, z^{\prime}\right)\right.\right. \\
& \left.-\boldsymbol{G}_{j}^{\prime}\left(z^{\prime}-0, z^{\prime}\right)\right\}+\boldsymbol{L}_{2}\left\{\frac{\partial}{\partial z} \boldsymbol{G}_{j}^{\prime}\left(z^{\prime}+0, z^{\prime}\right)\right. \\
& \left.\left.-\frac{\partial}{\partial z} \boldsymbol{G}_{j}^{\prime}\left(z^{\prime}-0, z^{\prime}\right)\right\}\right]
\end{aligned}
$$




$$
\begin{aligned}
& \times \delta\left(z-z^{\prime}\right)+\left[\boldsymbol{L}_{1} \boldsymbol{\Delta}_{j}+\boldsymbol{L}_{2}\left\{\boldsymbol{G}_{j}^{\prime}\left(z^{\prime}+0, z^{\prime}\right)-\boldsymbol{G}_{j}^{\prime}\left(z^{\prime}-0, z^{\prime}\right)\right\}\right] \\
& \times \frac{\partial}{\partial z} \delta\left(z-z^{\prime}\right)+\boldsymbol{L}_{2} \boldsymbol{\Delta}_{j} \frac{\partial^{2}}{\partial z^{2}} \delta\left(z-z^{\prime}\right)=-\boldsymbol{I}_{j} \delta\left(z-z^{\prime}\right), \quad(\mathrm{B} 8)
\end{aligned}
$$

where $\boldsymbol{I}_{j}$ is the $j$ th column of $\boldsymbol{I}$. By comparing the coefficients for the second $z$ derivative of the $\delta$ function on both sides of the equation, we obtain

$$
\Delta_{1 j}=\Delta_{2 j}=0,
$$

where $\Delta_{i j}$ denotes the $i$ th component of vector $\boldsymbol{\Delta}_{j}$. The quantities $G_{i j}^{\prime}$ and $I_{i j}$ are similarly defined as the $i$ th component of vector $\boldsymbol{G}_{j}^{\prime}$ and $\boldsymbol{I}_{j}$, respectively. By comparing the coefficients for the first $z$ derivative of the $\delta$ function, we find

$$
\begin{gathered}
i k_{x} \Delta_{3 j}+G_{1 j}^{\prime}\left(z^{\prime}+0, z^{\prime}\right)-G_{1 j}^{\prime}\left(z^{\prime}-0, z^{\prime}\right)=0, \\
G_{2 j}^{\prime}\left(z^{\prime}+0, z^{\prime}\right)-G_{2 j}^{\prime}\left(z^{\prime}-0, z^{\prime}\right)=0 .
\end{gathered}
$$

Finally by comparing the coefficients for the $\delta$ function, we obtain

$$
\begin{gathered}
k^{2}\left[\varepsilon_{h}\left(z^{\prime}\right)\right]_{x z} \Delta_{3 j}+i k_{x}\left\{G_{3 j}^{\prime}\left(z^{\prime}+0, z^{\prime}\right)-G_{3 j}^{\prime}\left(z^{\prime}-0, z^{\prime}\right)\right\} \\
+\left\{\frac{\partial}{\partial z} G_{1 j}^{\prime}\left(z^{\prime}+0, z^{\prime}\right)-\frac{\partial}{\partial z} G_{1 j}^{\prime}\left(z^{\prime}-0, z^{\prime}\right)\right\}=-I_{1 j}, \\
k^{2}\left[\boldsymbol{\varepsilon}_{h}\left(z^{\prime}\right)\right]_{y z} \Delta_{3 j}+\left\{\frac{\partial}{\partial z} G_{2 j}^{\prime}\left(z^{\prime}+0, z^{\prime}\right)-\frac{\partial}{\partial z} G_{2 j}^{\prime}\left(z^{\prime}-0, z^{\prime}\right)\right\} \\
=-I_{2 j}, \\
-k_{x}^{2} \Delta_{3 j}+k^{2}\left[\varepsilon_{h}\left(z^{\prime}\right)\right]_{z z} \Delta_{3 j}+i k_{x}\left\{G_{1 j}^{\prime}\left(z^{\prime}+0, z^{\prime}\right)\right. \\
\left.\quad-G_{1 j}^{\prime}\left(z^{\prime}-0, z^{\prime}\right)\right\}=k^{2}\left[\varepsilon_{h}\left(z^{\prime}\right)\right]_{z z} \Delta_{3 j}=-I_{3 j} .
\end{gathered}
$$

For the transformation in Eq. (B11c), Eq. (B10a) is used. From Eqs. (B9) and (B11c), $\boldsymbol{\Delta}_{j}$ can be found,

$$
\Delta_{i j}\left(z^{\prime}\right)= \begin{cases}-\frac{1}{k^{2} \varepsilon_{z z}^{\left(n^{\prime}\right)}} & \text { for } i=j=3 \\ 0 & \text { otherwise }\end{cases}
$$

where $n^{\prime}$ indicates the layer in which $z^{\prime}$ is involved. With the explicit expression for $\boldsymbol{E}_{l}$ 's, Eqs. (B10a), (B10b), (B11a), and (B11b) are transformed into

$$
Q\left(\begin{array}{lll}
g_{11} & g_{12} & g_{13} \\
g_{21} & g_{22} & g_{23} \\
g_{31} & g_{32} & g_{33} \\
g_{41} & g_{42} & g_{43}
\end{array}\right)=\left(\begin{array}{ccc}
0 & 0 & \frac{i k_{x}}{k^{2} \varepsilon_{z z}^{\left(n^{\prime}\right)}} \\
0 & 0 & 0 \\
-1 & 0 & \frac{\varepsilon_{x z}^{\left(n^{\prime}\right)}}{\varepsilon_{z z}^{\left(n^{\prime}\right)}} \\
0 & -1 & \frac{\varepsilon_{y z}^{\left(n^{\prime}\right)}}{\varepsilon_{z z}^{\left(n^{\prime}\right)}}
\end{array}\right) .
$$

Here $Q$ is a $4 \times 4$ matrix in the form

$$
\begin{gathered}
Q_{1 j}=\lambda_{j} b_{j \mu}^{\left(n^{\prime}\right)} e_{1 \mu}^{\left(n^{\prime}\right)} \exp \left\{i k_{\mu}^{\left(n^{\prime}\right)} \zeta^{\left(n^{\prime}\right)}\right\}, \\
Q_{2 j}=\lambda_{j} b_{j \mu}^{\left(n^{\prime}\right)} e_{2 \mu}^{\left(n^{\prime}\right)} \exp \left\{i k_{\mu}^{\left(n^{\prime}\right)} \zeta^{\left(n^{\prime}\right)}\right\}, \\
Q_{3 j}=i \lambda_{j} b_{j \mu}^{\left(n^{\prime}\right)}\left(k_{x} e_{3 \mu}^{\left(n^{\prime}\right)}+k_{\mu}^{\left(n^{\prime}\right)} e_{1 \mu}^{\left(n^{\prime}\right)}\right) \exp \left\{i k_{\mu}^{\left(n^{\prime}\right)} \zeta^{\left(n^{\prime}\right)}\right\}, \\
Q_{4 j}=i \lambda_{j} b_{j \mu}^{\left(n^{\prime}\right)} k_{\mu}^{\left(n^{\prime}\right)} e_{2 \mu}^{\left(n^{\prime}\right)} \exp \left\{i k_{\mu}^{\left(n^{\prime}\right)} \zeta^{\left(n^{\prime}\right)}\right\},
\end{gathered}
$$

where

$$
\lambda_{j}= \begin{cases}-1 & \text { for } j=1,2 \\ 1 & \text { for } j=3,4 .\end{cases}
$$

Summation on $\mu$ is required but not on $j$. By solving Eq. (B13) for $g_{l j}$, we obtain the Green's function $\boldsymbol{G}\left(z, z^{\prime} ; k_{x}\right)$ through Eqs. (B1), (B4), and (B12).

\section{APPENDIX C: REFLECTION AND TRANSMISSION COEFFICIENT FOR ACOUSTIC WAVES}

Equation (24) can be transformed into another convenient form

$$
\boldsymbol{A}_{s}=\boldsymbol{B}_{s} \boldsymbol{C}_{s},
$$

where $6 \times 6$ matrices $\boldsymbol{A}_{s}, \boldsymbol{B}_{s}$, and $\boldsymbol{C}_{s}$ are defined as

$$
\boldsymbol{A}_{s}=\left(\begin{array}{cccccc}
v_{1}^{(n)} u_{1 x}^{(n)} & v_{2}^{(n)} u_{2 x}^{(n)} & v_{3}^{(n)} u_{3 x}^{(n)} & v_{1}^{(n+1)} u_{1 x}^{(n+1)} & v_{2}^{(n+1)} u_{2 x}^{(n+1)} & v_{3}^{(n+1)} u_{3 x}^{(n+1)} \\
v_{1}^{(n)} u_{1 y}^{(n)} & v_{2}^{(n)} u_{2 y}^{(n)} & v_{3}^{(n)} u_{3 y}^{(n)} & v_{1}^{(n+1)} u_{1 y}^{(n+1)} & v_{2}^{(n+1)} u_{2 y}^{(n+1)} & v_{3}^{(n+1)} u_{3 y}^{(n+1)} \\
v_{1}^{(n)} u_{1 z}^{(n)} & v_{2}^{(n)} u_{2 z}^{(n)} & v_{3}^{(n)} u_{3 z}^{(n)} & v_{1}^{(n+1)} u_{1 z}^{(n+1)} & v_{2}^{(n+1)} u_{2 z}^{(n+1)} & v_{3}^{(n+1)} u_{3 z}^{(n+1)} \\
C_{x i}^{(n)} u_{1 i}^{(n)} & C_{x i}^{(n)} u_{2 i}^{(n)} & C_{x i}^{(n)} u_{3 i}^{(n)} & -C_{x i}^{(n+1)} u_{1 i}^{(n+1)} & -C_{x i}^{(n+1)} u_{2 i}^{(n+1)} & -C_{x i}^{(n+1)} u_{3 i}^{(n+1)} \\
C_{y i}^{(n)} u_{1 i}^{(n)} & C_{y i}^{(n)} u_{2 i}^{(n)} & C_{y i}^{(n)} u_{3 i}^{(n)} & -C_{y i}^{(n+1)} u_{1 i}^{(n+1)} & -C_{y i}^{(n+1)} u_{2 i}^{(n+1)} & -C_{y i}^{(n+1)} u_{3 i}^{(n+1)} \\
C_{z i}^{(n)} u_{1 i}^{(n)} & C_{z i}^{(n)} u_{2 i}^{(n)} & C_{z i}^{(n)} u_{3 i}^{(n)} & -C_{z i}^{(n+1)} u_{1 i}^{(n+1)} & -C_{z i}^{(n+1)} u_{2 i}^{(n+1)} & -C_{z i}^{(n+1)} u_{3 i}^{(n+1)}
\end{array}\right),
$$




$$
\begin{gathered}
\boldsymbol{B}_{s}=\left(\begin{array}{llllll}
v_{1}^{(n+1)} u_{1 x}^{(n+1)} & v_{2}^{(n+1)} u_{2 x}^{(n+1)} & v_{3}^{(n+1)} u_{3 x}^{(n+1)} & v_{1}^{(n)} u_{1 x}^{(n)} & v_{2}^{(n)} u_{2 x}^{(n)} & v_{3}^{(n)} u_{3 x}^{(n)} \\
v_{1}^{(n+1)} u_{1 y}^{(n+1)} & v_{2}^{(n+1)} u_{2 y}^{(n+1)} & v_{3}^{(n+1)} u_{3 y}^{(n+1)} & v_{1}^{(n)} u_{1 y}^{(n)} & v_{2}^{(n)} u_{2 y}^{(n)} & v_{3}^{(n)} u_{3 y}^{(n)} \\
v_{1}^{(n+1)} u_{1 z}^{(n+1)} & v_{2}^{(n+1)} u_{2 z}^{(n+1)} & v_{3}^{(n+1)} u_{3 z}^{(n+1)} & v_{1}^{(n)} u_{1 z}^{(n)} & v_{2}^{(n)} u_{2 z}^{(n)} & v_{3}^{(n)} u_{3 z}^{(n)} \\
C_{x i}^{(n+1)} u_{1 i}^{(n+1)} & C_{x i}^{(n+1)} u_{2 i}^{(n+1)} & C_{x i}^{(n+1)} u_{3 i}^{(n+1)} & -C_{x i}^{(n)} u_{1 i}^{(n)} & -C_{x i}^{(n)} u_{2 i}^{(n)} & -C_{x i}^{(n)} u_{3 i}^{(n)} \\
C_{y i}^{(n+1)} u_{1 i}^{(n+1)} & C_{y i}^{(n+1)} u_{2 i}^{(n+1)} & C_{y i}^{(n+1)} u_{3 i}^{(n+1)} & -C_{y i}^{(n)} u_{1 i}^{(n)} & -C_{y i}^{(n)} u_{2 i}^{(n)} & -C_{y i}^{(n)} u_{3 i}^{(n)} \\
C_{z i}^{(n+1)} u_{1 i}^{(n+1)} & C_{z i}^{(n+1)} u_{2 i}^{(n+1)} & C_{z i}^{(n+1)} u_{3 i}^{(n+1)} & -C_{z i}^{(n)} u_{1 i}^{(n)} & -C_{z i}^{(n)} u_{2 i}^{(n)} & -C_{z i}^{(n)} u_{3 i}^{(n)}
\end{array}\right), \\
\boldsymbol{C}_{s}=\left(\begin{array}{llllll}
t_{11} & t_{21} & t_{31} & r_{11}^{\prime} & r_{21}^{\prime} & r_{31}^{\prime} \\
t_{12} & t_{22} & t_{32} & r_{12}^{\prime} & r_{22}^{\prime} & r_{32}^{\prime} \\
t_{13} & t_{23} & t_{33} & r_{13}^{\prime} & r_{23}^{\prime} & r_{33}^{\prime} \\
r_{11} & r_{21} & r_{31} & t_{11}^{\prime} & t_{21}^{\prime} & t_{31}^{\prime} \\
r_{12} & r_{22} & r_{32} & t_{12}^{\prime} & t_{22}^{\prime} & t_{32}^{\prime} \\
r_{13} & r_{23} & r_{33} & t_{13}^{\prime} & t_{23}^{\prime} & t_{33}^{\prime}
\end{array}\right)
\end{gathered}
$$

Here $C_{i j}^{(n)}(i, j=x, y, z)$ are the components of $\boldsymbol{C}^{(n)}$ in Eq. (17), and $u_{\mu i}^{(n)}$ is the $i$ th component of displacement vector $\boldsymbol{u}_{\mu}^{(n)}$ (mode $\mu$ in layer $n$ ). In this form the transmittivity and reflectivity for strain at the interface is given as follows: $t_{\mu \nu}$, transmittivity from mode $\mu$ in the $n$th layer to mode $\nu$ in the $(n+1)$ th layer; $r_{\mu \nu}$, reflectivity from mode $\mu$ in the $n$th layer to mode $\nu$ in the $n$th layer; $t_{\mu \nu}^{\prime}$, transmittivity from mode $\mu$ in the $(n+1)$ th layer to mode $\nu$ in the $n$th layer; and $r_{\mu \nu}^{\prime}$, reflectivity from mode $\mu$ in the $(n+1)$ th layer to mode $\nu$ in the $(n+1)$ th layer.

${ }^{1}$ C. Thomsen, H. T. Grahn, H. J. Maris, and J. Tauc, Phys. Rev. B 34, 4129 (1986).

${ }^{2}$ O. B. Wright and K. Kawashima, Phys. Rev. Lett. 69, 1668 (1992).

${ }^{3}$ B. Perrin, B. Bonello, J. C. Jeannet, and E. Romatet, Prog. Nat. Sci. S6, S444 (1996).

${ }^{4}$ C. J. K. Richardson, M. J. Ehrlich, and J. W. Wagner, J. Opt. Soc. Am. B 16, 1007 (1999).

${ }^{5}$ D. H. Hurley and O. B. Wright, Opt. Lett. 24, 1305 (1999).

${ }^{6}$ C. K. Sun, J. C. Liang, and X. Y. Yu, Phys. Rev. Lett. 84, 179 (2000).

${ }^{7}$ A. Devos and C. Lerouge, Phys. Rev. Lett. 86, 2669 (2001).

${ }^{8}$ T. Saito, O. Matsuda, and O. B. Wright, Phys. Rev. B 67, 205421 (2003).

${ }^{9}$ O. Matsuda, T. Tachizaki, T. Fukui, J. J. Baumberg, and O. B. Wright, Phys. Rev. B 71, 115330 (2005).

${ }^{10}$ A. Huynh, N. D. Lanzillotti-Kimura, B. Jusserand, B. Perrin, A. Fainstein, M. F. Pascual-Winter, E. Peronne, and A. Lemaître, Phys. Rev. Lett. 97, 115502 (2006).

${ }^{11}$ A. V. Akimov, A. V. Scherbakov, D. R. Yakovlev, C. T. Foxon, and M. Bayer, Phys. Rev. Lett. 97, 037401 (2006).

${ }^{12}$ M. F. Pascual Winter, G. Rozas, A. Fainstein, B. Jusserand, B. Perrin, A. Huynh, P. O. Vaccaro, and S. Saravanan, Phys. Rev. Lett. 98, 265501 (2007).

${ }^{13}$ D. H. Hurley, O. B. Wright, O. Matsuda, V. E. Gusev, and O. V. Kolosov, Ultrasonics 38, 470 (2000).

${ }^{14}$ O. Matsuda, O. B. Wright, D. H. Hurley, V. E. Gusev, and K. Shimizu, Phys. Rev. Lett. 93, 095501 (2004).

${ }^{15}$ R. N. Kini, A. J. Kent, N. M. Stanton, and M. Henini, Appl. Phys. Lett. 88, 134112 (2006).

${ }^{16}$ T. Pezeril, N. Chigarev, P. Ruello, S. Gougeon, D. Mounier,
J.-M. Breteau, P. Picart, and V. Gusev, Phys. Rev. B 73, 132301 (2006).

${ }^{17}$ T. Pezeril, P. Ruello, S. Gougeon, N. Chigarev, D. Mounier, J. M. Breteau, P. Picart, and V. Gusev, Phys. Rev. B 75, 174307 (2007).

${ }^{18}$ C. Rossignol, J. M. Rampnoux, M. Perton, B. Audoin, and S. Dilhaire, Phys. Rev. Lett. 94, 166106 (2005).

${ }^{19}$ T. Dehoux, N. Chigarev, C. Rossignol, and B. Audoin, Phys. Rev. B 76, 024311 (2007).

${ }^{20}$ O. B. Wright, J. Appl. Phys. 71, 1617 (1992).

${ }^{21}$ O. Matsuda and O. B. Wright, J. Opt. Soc. Am. B 19, 3028 (2002).

${ }^{22}$ A. A. Maradudin and D. L. Mills, Phys. Rev. B 11, 1392 (1975).

${ }^{23}$ K. R. Subbaswamy and A. A. Maradudin, Phys. Rev. B 18, 4181 (1978).

${ }^{24}$ B. A. Auld, Acoustic Fields and Waves in Solids, 2nd ed. (Krieger, Florida, 1990).

${ }^{25}$ T. C. T. Ting, Anisotropic Elasticity (Oxford University Press, New York, 1996).

${ }^{26}$ J. F. Nye, Physical Properties of Crystals (Oxford University Press, Oxford, 1957).

${ }^{27}$ D. F. Nelson and M. Lax, Phys. Rev. Lett. 24, 379 (1970).

${ }^{28}$ D. F. Nelson and M. Lax, Phys. Rev. B 3, 2778 (1971).

${ }^{29}$ Y. Liu and A. H. W. Ngan, Scr. Mater. 44, 237 (2001).

${ }^{30}$ H. N. Lin, R. J. Stoner, H. J. Maris, and J. Tauc, J. Appl. Phys. 69, 3816 (1991).

${ }^{31}$ O. L. Anderson, in Physical Acoustics, edited by W. P. Mason (Academic, New York, 1965), Vol. 3B, chap. 2, pp. 43-95.

32 J. Kushibiki, H. Maehara, and N. Chubachi, J. Appl. Phys. 53, 5509 (1982).

${ }^{33}$ M. Rothenfusser, W. Dietsche, and H. Kinder, in Phonon Scat- 
tering in Condensed Matter, Springer Series in Solid-State Sciences Vol. 51, edited by W. Eisenmenger, K. Lassmann, and S. Döttinger (Springer, New York, 1984), p. 419.

${ }^{34}$ R. Vacher and L. Boyer, Phys. Rev. B 6, 639 (1972).

${ }^{35}$ H. Z. Cummins and P. E. Schoen, in Laser Handbook, edited by F. T. Arecchi and E. O. Schulz-Dubois (North-Holland, Amsterdam, 1972), Vol. 2, chap. E1, pp. 1029-1075.

${ }^{36}$ O. Matsuda and O. B. Wright, Anal. Sci. 17, S216 (2001).

${ }^{37}$ O. B. Wright, Phys. Rev. B 49, 9985 (1994).

${ }^{38}$ S. I. Anisimov, B. L. Kapeliovich, and T. L. Perel'man, Sov. Phys. JETP 39, 375 (1974).

${ }^{39}$ P. B. Corkum, F. Brunel, N. K. Sherman, and T. Srinivasan-Rao, Phys. Rev. Lett. 61, 2886 (1988).

${ }^{40}$ V. E. Gusev and O. B. Wright, Phys. Rev. B 57, 2878 (1998).

${ }^{41}$ C. A. Cinnamon, Phys. Rev. 46, 215 (1934).

${ }^{42}$ CRC Handbook of Chemistry and Physics, 85 th ed., edited by D. R. Lide (CRC, Boca Raton, FL, 2004).

${ }^{43}$ C. Kittel, Introduction to Solid State Physics, 6th ed. (Wiley, New York, 1986).

${ }^{44}$ S. Kashiwada, O. Matsuda, J. J. Baumberg, R. L. Voti, and O. B. Wright, J. Appl. Phys. 100, 073506 (2006).

${ }^{45}$ R. W. Dixon, J. Appl. Phys. 38, 5149 (1967)

${ }^{46}$ K. Postava, M. Aoyama, T. Yamaguchi, and H. Oda, Appl. Surf. Sci. 175-176, 276 (2001).

${ }^{47}$ R. Vacher and J. Pelous, J. Non-Cryst. Solids 45, 397 (1981).

${ }^{48}$ M. Hase, K. Ishioka, J. Demsar, K. Ushida, and M. Kitajima, Phys. Rev. B 71, 184301 (2005).

${ }^{49}$ M. Hase, K. Ishioka, J. Demsar, K. Ushida, and M. Kitajima, Phys. Rev. B 72, 189902(E) (2005).

${ }^{50}$ P. J. Lin-Chung and S. Teitler, J. Opt. Soc. Am. A 1, 703 (1984).

${ }^{51}$ R. S. Weis and T. K. Gaylord, J. Opt. Soc. Am. A 4, 1720 (1987).

${ }^{52}$ C. J. Chen, A. Lien, and M. I. Mathan, J. Opt. Soc. Am. A 14, 3125 (1997).

${ }^{53}$ O. Matsuda and C. Glorieux, J. Acoust. Soc. Am. 121, 3437 (2007).

${ }^{54}$ We use the word scattering also in the case of optical reflection, since the optical scattering from an interface or from a small change in refractive index have a similar origin, i.e., an inhomogeneity in the electrical permittivity.

${ }^{55} \mathrm{We}$ assume that there is no free electric charge and no conduction current.

${ }^{56}$ The cut angle in Ref. 14 was stated erroneously. This does not affect the conclusions given in Ref. 14 .

${ }^{57}$ The insets involving $45^{\circ}$ shown in Fig. 1 of Ref. 14 were erroneously depicted.

${ }^{58}$ The 200 ps window results in a Fourier transform line width of $\sim 5 \mathrm{GHz}$. However, our qualitative analysis does not use the Fourier transform, and is not affected by this broadening.

${ }^{59}$ Our experiment represents a time-domain measurement of Brillouin scattering caused by coherent phonons, whereas conventional Brillouin scattering measurements are done in the frequency domain with scattering caused by incoherent phonons.

${ }^{60}$ The criterion for a significant contribution from the acoustic sources for times $t \gtrsim \tau_{e}$ is that the effect of thermal diffusion should be supersonic (Ref. 1). This can be expressed by the condition $z_{e}^{2} / D_{e} \lesssim z_{e} / v$, where $D_{e} \equiv \kappa / C_{e}$ is the thermal diffusivity and $v$ is the sound velocity of the relevant acoustic mode. Another way of writing this inequality is $D_{e} /\left(v z_{e}\right) \geq 1$. In the case of zinc at $600 \mathrm{~K}, D_{e} / v z_{e} \sim 0.7$ for quasishear waves and $D_{e} / v z_{e} \sim 0.3$ for quasilongitudinal waves.

${ }^{61}$ The shape of the shear pulses was erroneously given in Ref. 14 because of an error in sign.

${ }^{62}$ The minus signs on the right-hand side of the third and forth equations are the consequence of our choice of the direction of the $45^{\circ}$ polarization: $(1 / 2,-1 / \sqrt{2},-1 / 2)$. If instead $(1 / 2,1 / \sqrt{2},-1 / 2)$ is used, all these minus signs would be replaced with plus signs.

${ }^{63}$ The expression for reflectivity change assumed in Ref. 17 only involves the photoelastic effect and so the absolute values of the photoelastic constants could not be derived.

${ }^{64}$ Reference 45 gives the photoelastic constant $p_{i j k l}$ defined for the impermeability $B_{i j}=1 / \varepsilon_{0}\left(\partial E_{i} / \partial D_{j}\right)$ according to $\Delta B_{i j}=p_{i j k l} \eta_{k l}$. The relation $P_{i j k l}=-\varepsilon^{2} p_{i j k l}$ holds for isotropic media. 